TRANSACTIONS OF THE

AMERICAN MATHEMATICAL SOCIETY

Volume 365, Number 1, January 2013, Pages 143-160

S 0002-9947(2012)05531-0

Article electronically published on July 25, 2012

\title{
CRITICAL CONES OF CHARACTERISTIC VARIETIES
}

\author{
ROBERTO BOLDINI
}

\begin{abstract}
Let $M$ be a left module over a Weyl algebra in characteristic zero. Given natural weight vectors $\nu$ and $\omega$, we show that the characteristic varieties arising from filtrations with weight vector $\nu+s \omega$ stabilize to a certain variety determined by $M, \nu, \omega$ as soon as the natural number $s$ grows beyond a bound which depends only on $M$ and $\nu$ but not on $\omega$.

As a consequence, in the notable case when $\nu$ is the standard weight vector, these characteristic varieties deform to the critical cone of the $\omega$-characteristic variety of $M$ as soon as $s$ grows beyond an invariant of $M$.

As an application, we give a new, easy, non-homological proof of a classical result, namely, that the $\omega$-characteristic varieties of $M$ all have the same Krull dimension.

The set of all $\omega$-characteristic varieties of $M$ is finite. We provide an upper bound for its cardinality in terms of supports of universal Gröbner bases in the case when $M$ is cyclic. By the above stability result, we conjecture a second upper bound in terms of total degrees of universal Gröbner bases and of Fibonacci numbers in the case when $M$ is cyclic over the first Weyl algebra.
\end{abstract}

\section{INTRODUCTION}

Let $n \in \mathbb{N}$, let $W$ be the $n^{\text {th }}$ Weyl algebra over a field $K$ of characteristic 0 , and let $\Omega=\left\{\omega \in \mathbb{N}_{0}^{2 n} \mid \omega_{i}+\omega_{i+n}>0\right.$ for $\left.1 \leq i \leq n\right\}$. For each $\omega \in \Omega$ consider the $\omega$-degree filtration $\mathrm{F}^{\omega} W=\left(\mathrm{F}_{i}^{\omega} W\right)_{i \in \mathbb{Z}}$ of $W$ and any good $\mathrm{F}^{\omega} W$-filtration $\mathrm{F}^{\omega} M=\left(\mathrm{F}_{i}^{\omega} M\right)_{i \in \mathbb{Z}}$ of a left $W$-module $M$. We construct $\mathrm{G}^{\omega} W=\bigoplus_{i \in \mathbb{Z}} \mathrm{F}_{i}^{\omega} W / \mathrm{F}_{i-1}^{\omega} W$ and $\mathrm{G}^{\omega} M=$ $\bigoplus_{i \in \mathbb{Z}} \mathrm{F}_{i}^{\omega} M / \mathrm{F}_{i-1}^{\omega} M$. Then $\mathrm{G}^{\omega} W$ is a ring canonically isomorphic to the commutative polynomial ring $K[X, Y]$ in the indeterminates $X_{1}, \ldots, X_{n}$ and $Y_{1}, \ldots, Y_{n}$, and $\mathrm{G}^{\omega} M$ is a finitely generated $K[X, Y]$-module. For a fixed $\omega \in \Omega$, the radical ideal $\sqrt{ }\left(0: \mathrm{G}^{\omega} M\right)$ of $K[X, Y]$ is independent of the choice of a good $\mathrm{F}^{\omega} W$-filtration $\mathrm{F}^{\omega} M$ of $M$. So we may define the $\omega$-characteristic variety of $M$ as the closed subset $\mathcal{V}^{\omega}(M)=\operatorname{Var}\left(0: \mathrm{G}^{\omega} M\right)$ of $\operatorname{Spec}(K[X, Y])$.

Similarly, we consider the $\nu$-degree filtrations $\mathrm{F}^{\nu} K[X, Y]$ of $K[X, Y], \nu \in \mathbb{N}_{0}^{2 n}$, and good $\mathrm{F}^{\nu} K[X, Y]$-filtrations $\mathrm{F}^{\nu} N$ of $K[X, Y]$-modules $N$ and construct the rings $\mathrm{G}^{\nu} K[X, Y]$, canonically isomorphic to $K[X, Y]$, and the finitely generated $K[X, Y]$ modules $\mathrm{G}^{\nu} N$. Again, for a fixed $\nu \in \mathbb{N}_{0}^{2 n}$, the radical ideal $\sqrt{ }\left(0: \mathrm{G}^{\nu} N\right)$ of $K[X, Y]$ does not depend on the choice of a good $\mathrm{F}^{\nu} K[X, Y]$-filtration $\mathrm{F}^{\nu} N$ of $N$.

Received by the editors July 21, 2010 and, in revised form, December 4, 2010, and December $24,2010$.

2010 Mathematics Subject Classification. Primary 13C15, 13N10, 13P10, 16P90, 16 W70.

The author thanks Professor Markus Brodmann and Professor Joseph Ayoub, University of Zurich.

(C)2012 American Mathematical Society Reverts to public domain 28 years from publication 
The main result of this paper is that for each $\nu \in \mathbb{N}_{0}^{2 n}$ there exists $s_{0} \in \mathbb{N}_{0}$ such that for all $s \in \mathbb{N}$ with $s>s_{0}$ and all $\omega \in \Omega$ in $K[X, Y]$ it holds

$$
\sqrt{ }\left(0: \mathrm{G}^{\nu} \mathrm{G}^{\omega} M\right)=\sqrt{ }\left(0: \mathrm{G}^{\nu+s \omega} M\right) .
$$

Observe that $s_{0}$ does not depend on $\omega$. We can choose the lowest such $s_{0}$ in $\mathbb{N}_{0}$, denoted $\kappa_{\nu}(M)$. If $L$ is a left ideal of $W$, we give an upper bound for $\kappa_{\nu}(W / L)$ in terms of total degrees of elements of universal Gröbner bases of $L$, more precisely,

$$
\kappa_{\nu}(W / L) \leq \gamma_{\nu}(L)
$$

where

$$
\gamma_{\nu}(L)=\inf _{U} \sup _{u \in U} \operatorname{deg}^{\nu}(u)
$$

the infimum being taken over all universal Gröbner bases $U$ of $L$.

A case with evident geometrical meaning is when $\nu=(1)=(1, \ldots, 1) \in \mathbb{N}_{0}^{2 n}$. Equality (A) says that the "affine deformations" $\mathcal{V}^{(1)+s \omega}(M)$ of $\mathcal{V}^{\omega}(M)$ stabilize for large $s$ to the critical cone $\mathcal{C}^{\omega}(M)=\operatorname{Var}\left(0: \mathrm{G}^{(1)} \mathrm{G}^{\omega} M\right)$ of $\mathcal{V}^{\omega}(M)$. Thus the minimal limit beyond which this occurs, namely, $\kappa(M)=\kappa_{(1)}(M)$, is - surprisinglyan invariant of $M$. Upper bounds for the greatest total degree of Gröbner bases and of reduced Gröbner bases of a left ideal $L$ of $W$ are given in [1] in terms of greatest total degrees of systems of generators of $L$, and hence, combining both results, we obtain an upper bound for $\kappa(W / L)$ also in such terms.

The critical cone $C$ of an affine variety $V \subseteq \mathbb{A}^{r}$ over an algebraically closed field $F$ is the cone with vertex at the origin $O \in \mathbb{A}^{r}$ tangent to $V$ at infinity. In other words, $C$ consists of all lines through $O$ along whose directions $V$ goes to infinity. To construct $C$, we choose an injection $\iota: \mathbb{A}^{r} \longmapsto \mathbb{P}^{r}$ of the affine space $\mathbb{A}^{r}$ into the projective space $\mathbb{P}^{r}$ over $F$ and put

$$
C=\iota^{-1}\left(\bigcup_{P \in \overline{\iota(V)} \backslash \iota(V)} \ell_{P}\right),
$$

where $\overline{\iota(V)}$ is the projective closure of $\iota(V)$ in $\mathbb{P}^{r}$ and $\ell_{P}$ is the projective line through the points $\iota(O)$ and $P$. One has that $C$ does not depend on the choice of $\iota$. Algebraically, if $I$ is any ideal of $F\left[Z_{1}, \ldots, Z_{r}\right]$ that defines $V$, then $C$ is defined by the ideal $J$ generated by the homogeneous components of greatest total degree of the polynomials in $I$, that is, $J$ is the leading form ideal of $I$ by total degree. Again, $C$ does not depend on the choice of $I$.

As a further consequence of equality (A), we are able to give an easy proof that $\operatorname{Kdim}_{K[X, Y]} \mathrm{G}^{\omega} M=\mathrm{GKdim}_{W} M$ for all $\omega \in \Omega$. Thus, without having to appeal to sophisticated homological methods as in classical proofs, we have shown in particular that the characteristic varieties $\mathcal{V}^{\omega}(M), \omega \in \Omega$, all have the same Krull dimension. The key point is that (A) allows us to pass from non-finite to finite filtrations, and the Gelfand-Kirillov dimension behaves well with finite discrete filtrations: $\mathrm{GKdim}_{\mathrm{G}^{\omega} W} \mathrm{G}^{\omega} M=\mathrm{GKdim}_{W} M$ whenever $\mathrm{F}^{\omega} M$ is finite and discrete. The second point is that the Gelfand-Kirillov dimension and Krull dimension agree in the category of finitely generated modules over any fixed finitely generated algebra over a field.

Fixing a left ideal $L$ of $W$, we give an upper bound for the number $\chi(L)$ of distinct ideals $\mathrm{G}^{\omega} L, \omega \in \Omega$, and hence of distinct $\omega$-characteristic varieties of $W / L$, namely,

$$
\chi(L) \leq \inf _{U} \prod_{u \in U} \sum_{0 \leq k \leq \# \operatorname{supp}(u)}\left(\begin{array}{c}
\# \operatorname{supp}(u) \\
k
\end{array}\right)
$$


the infimum being taken over all universal Gröbner bases of $L$. Equality (A) lets us conjecture a second upper bound in the case when $W$ is the first Weyl algebra, namely,

$$
\chi(L) \leq 2^{1+\gamma(L)}+1,
$$

where $\gamma(L)=\gamma_{(1)}(L)$. As mentioned before, in [1] one has an upper bound for $\gamma(L)$ in terms of total degrees of generators of $L$.

In Section 1 we recall some known facts about filtered rings and modules as well as their associated graded rings and modules.

In Section 2 we introduce Weyl algebras and state some of their basic properties, which are a generalization of results that can be found, for instance, in 8 . The proofs remain very similar, and we omit them here.

Section 3 is about Gröbner bases in Weyl algebras. Here, too, we recall known facts, important in the next section, in particular the existence of universal Gröbner bases for left ideals, and a very tight relation between the Gröbner bases of $\omega$-filtered left ideals and the Gröbner bases of their associated graded ideals.

In Section 4 we define $\omega$-characteristic varieties of a left $W$-module $M$ as some particular affine spectra, and not as algebraic zero sets, as is usual, for there is no reason here to work only over algebraically closed fields. Then we prove our main result (A) about the defining annihilators of such varieties.

In Section 5 we apply (A) to give an easy proof of the known result that the $\omega$ characteristic varieties of $M$ all have the same Gelfand-Kirillov and Krull dimension as $\omega$ varies in $\Omega$, namely, equal to the Gelfand-Kirillov dimension of $M$.

Finally in Section 6 we perform a computer experiment in order to try to classify the $\omega$-characteristic varieties of $M$ in the case when $M=W / L$ for a left ideal $L$ of $W$. This experiment lets us conjecture an upper bound for their number, namely (D).

\section{Filtrations And GRADings}

In this section we give a small review on filtered rings and modules and their associated graded objects. Most of this material can be found in or inferred from the books of Constantin Năstăsescu, Freddy van Oystaeyen, and Huishi Li, among which we particularly appreciate [13. Besides giving a very short proof of Proposition 1.26, we provide a proof of Proposition 1.28 and Remark 1.29, too, which we did not find in the literature.

Definition 1.1. A filtration $\mathcal{R}$ of a ring $R$ is a family $\left(\mathrm{F}_{i} \mathcal{R}\right)_{i \in \mathbb{Z}}$ of additive subgroups $\mathrm{F}_{i} \mathcal{R}$ of $R$ enjoying the following properties:

(a) $R=\bigcup_{i \in \mathbb{Z}} \mathrm{F}_{i} \mathcal{R}$

(b) $\mathrm{F}_{i-1} \mathcal{R} \subseteq \mathrm{F}_{i} \mathcal{R}$,

(c) $r \in \mathrm{F}_{i} \mathcal{R} \wedge s \in \mathrm{F}_{j} \mathcal{R} \Rightarrow r s \in \mathrm{F}_{i+j} \mathcal{R}$,

(d) $i<0 \Rightarrow \mathrm{F}_{i} \mathcal{R}=0$,

(e) $1 \in \mathrm{F}_{0} \mathcal{R}$,

so that $\mathrm{F}_{0} \mathcal{R}$ is a subring of $R$ and each $\mathrm{F}_{i} \mathcal{R}$ is a left $\mathrm{F}_{0} \mathcal{R}$-submodule of $R$.

If the ring $R$ is provided with a filtration $\mathcal{R}$, we say that the ordered pair $(R, \mathcal{R})$ is a filtered ring.

Let $(R, \mathcal{R})$ and $(S, \mathcal{S})$ be filtered rings. A homomorphism of $(R, \mathcal{R})$ in $(S, \mathcal{S})$ is a ring homomorphism $\phi$ of $R$ in $S$ such that $\phi\left(\mathrm{F}_{i} \mathcal{R}\right) \subseteq \mathrm{F}_{i} \mathcal{S}$. 
Definition 1.2. Let $(R, \mathcal{R})$ be a filtered ring. An $\mathcal{R}$-filtration $\mathcal{M}$ of a left $R$ module $M$ is a family $\left(\mathrm{F}_{i} \mathcal{M}\right)_{i \in \mathbb{Z}}$ of additive subgroups $\mathrm{F}_{i} \mathcal{M}$ of $M$ with the following properties:

(a) $M=\bigcup_{i \in \mathbb{Z}} \mathrm{F}_{i} \mathcal{M}$,

(b) $\mathrm{F}_{i-1} \mathcal{M} \subseteq \mathrm{F}_{i} \mathcal{M}$,

(c) $r \in \mathrm{F}_{i} \mathcal{R} \wedge m \in \mathrm{F}_{j} \mathcal{M} \Rightarrow r m \in \mathrm{F}_{i+j} \mathcal{M}$,

so that each $\mathrm{F}_{i} \mathcal{M}$ is a left $\mathrm{F}_{0} \mathcal{R}$-submodule of $M$.

If the left $R$-module $M$ is provided with an $\mathcal{R}$-filtration $\mathcal{M}$, we say that the ordered pair $(M, \mathcal{M})$ is an $\mathcal{R}$-filtered left $R$-module or simply a left $(R, \mathcal{R})$-module. Observe that a filtered ring is also a filtered left module over itself.

Let $(M, \mathcal{M})$ and $(N, \mathcal{N})$ be left $(R, \mathcal{R})$-modules. An $(R, \mathcal{R})$-homomorphism of $(M, \mathcal{M})$ in $(N, \mathcal{N})$ is a left $R$-module homomorphism $\phi$ of $M$ in $N$ such that $\phi\left(\mathrm{F}_{i} \mathcal{M}\right) \subseteq \mathrm{F}_{i} \mathcal{N}$.

Definition 1.3. Let $(R, \mathcal{R})$ be a filtered ring and $(M, \mathcal{M})$ be a left $(R, \mathcal{R})$-module. Let $N$ be a left $R$-submodule of $M$. There exist canonically induced $\mathcal{R}$-filtrations $\mathcal{N}=\left(\mathrm{F}_{i} \mathcal{M} \cap N\right)_{i \in \mathbb{Z}}$ of $N$ and $\mathcal{M} / \mathcal{N}=\left(\mathrm{F}_{i} \mathcal{M}+N / N\right)_{i \in \mathbb{Z}}$ of $M / N$. In this situation we call $(N, \mathcal{N})$ a submodule of $(M, \mathcal{M})$ and $(M / N, \mathcal{M} / \mathcal{N})$ a quotient module of $(M, \mathcal{M})$. Similarly, if $I$ is a left ideal of $R$ and $\mathcal{I}$ is the induced $\mathcal{R}$-filtration of $I$, we say that $(I, \mathcal{I})$ is a left ideal of $(R, \mathcal{R})$.

Definition 1.4. Let $(R, \mathcal{R})$ be a filtered ring. The associated graded ring $\mathrm{G} \mathcal{R}$ of $R$ with respect to $\mathcal{R}$ is the commutative group $\bigoplus_{i \in \mathbb{Z}} \mathrm{F}_{i} \mathcal{R} / \mathrm{F}_{i-1} \mathcal{R}$ provided with a multiplication given by $\left(r_{i}+\mathrm{F}_{i-1} \mathcal{R}\right)_{i \in \mathbb{Z}}\left(s_{j}+\mathrm{F}_{j-1} \mathcal{R}\right)_{j \in \mathbb{Z}}=\left(\sum_{i+j=k} r_{i} s_{j}+\mathrm{F}_{k-1} \mathcal{R}\right)_{k \in \mathbb{Z}}$, which indeed turns $\mathrm{G} \mathcal{R}$ into a ring.

Let $(M, \mathcal{M})$ be a left $(R, \mathcal{R})$-module. The associated graded left $\mathrm{G} \mathcal{R}$-module $\mathrm{G} \mathcal{M}$ of $M$ with respect to $\mathcal{M}$ is the commutative group $\bigoplus_{i \in \mathbb{Z}} \mathrm{F}_{i} \mathcal{M} / \mathrm{F}_{i-1} \mathcal{M}$ with a $\mathrm{G} \mathcal{R}$ action defined by $\left(r_{i}+\mathrm{F}_{i-1} \mathcal{R}\right)_{i \in \mathbb{Z}}\left(m_{j}+\mathrm{F}_{j-1} \mathcal{M}\right)_{j \in \mathbb{Z}}=\left(\sum_{i+j=k} r_{i} m_{j}+\mathrm{F}_{k-1} \mathcal{M}\right)_{k \in \mathbb{Z}}$, which indeed turns GM into a left $\mathrm{G} \mathcal{R}$-module.

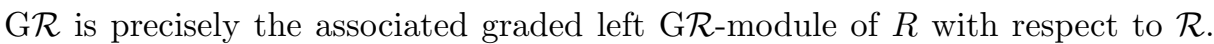
We denote the $i^{\text {th }}$ homogeneous component $\mathrm{F}_{i} \mathcal{M} / \mathrm{F}_{i-1} \mathcal{M}$ of $\mathrm{G} \mathcal{M}$ by $\mathrm{G}_{i} \mathcal{M}$. Then $\mathrm{G}_{0} \mathcal{R}$ is a subring of $\mathrm{G} \mathcal{R}$ and each $\mathrm{G}_{i} \mathcal{M}$ is a left $\mathrm{G}_{0} \mathcal{R}$-submodule of GM.

Remark 1.5. Let $(R, \mathcal{R})$ be a filtered ring, $(X, \mathcal{X})$ and $(Y, \mathcal{Y})$ be left $(R, \mathcal{R})$-modules, and $\phi$ be a homomorphism of $(X, \mathcal{X})$ in $(Y, \mathcal{Y})$. We have canonical $\mathrm{F}_{0} \mathcal{R}$-module homomorphisms $\mathrm{F}_{i} \mathcal{X} / \mathrm{F}_{i-1} \mathcal{X} \rightarrow \mathrm{F}_{i} \mathcal{Y} / \mathrm{F}_{i-1} \mathcal{Y}$ whose direct sum is a graded left $\mathrm{G} \mathcal{R}$ module homomorphism $\mathrm{GX} \rightarrow \mathrm{G} \mathcal{Y}$.

If $(N, \mathcal{N}) \underset{\pi}{\longrightarrow}(M, \mathcal{M}) \rightarrow(P, \mathcal{P})$ is a strict exact sequence of $(R, \mathcal{R})$-modules, that is, $N \stackrel{\nu}{\longmapsto} M \stackrel{\pi}{\rightarrow} P$ is an exact sequence of $R$-modules with $\nu\left(\mathrm{F}_{i} \mathcal{N}\right)=\mathrm{F}_{i} \mathcal{M} \cap \operatorname{Im}(\nu)$ and $\pi\left(\mathrm{F}_{i} \mathcal{M}\right)=\mathrm{F}_{i} \mathcal{P} \cap \operatorname{Im}(\pi)$, then there is an exact sequence $\mathrm{G} \mathcal{N} \mapsto \mathrm{G} \mathcal{M} \rightarrow \mathrm{GP}$ of graded left $\mathrm{G} \mathcal{R}$-modules.

In particular, if $(N, \mathcal{N})$ is a submodule of $(M, \mathcal{M})$ and $(M / N, \mathcal{M} / \mathcal{N})$ is a quotient module of $(M, \mathcal{M})$, then we obtain an exact sequence $\mathrm{G} \mathcal{N} \longmapsto \mathrm{G} \mathcal{M} \rightarrow \mathrm{G} \mathcal{M} / \mathcal{N}$, so that $\mathrm{G} \mathcal{M} / \mathcal{N} \cong \mathrm{G} \mathcal{M} / \mathrm{G} \mathcal{N}$ as graded left $\mathrm{G} \mathcal{R}$-modules.

Remark 1.6. Let $(R, \mathcal{R})$ be a filtered ring, $(M, \mathcal{M})$ be a left $(R, \mathcal{R})$-module, and $(N, \mathcal{N})$ be a submodule of $(M, \mathcal{M})$. By Remark 1.5 we may write $\mathrm{G} \mathcal{N} \subseteq \mathrm{G} \mathcal{M}$.

Assume that $N \subsetneq M$. Then the set $I=\left\{i \in \mathbb{Z} \mid \mathrm{F}_{i} \mathcal{M} \nsubseteq N\right\}$ is non-empty. Assume further that the $\mathcal{R}$-filtration $\mathcal{M}$ is discrete, that is, $\mathrm{F}_{i} \mathcal{M}=0$ for $i \ll 0$. Then $I$ admits a unique least element $i_{0}$. Suppose that $\mathrm{G} \mathcal{N}=$ GM. Then $\mathrm{G} \mathcal{M} / \mathcal{N} \cong \mathrm{G} \mathcal{M} / \mathrm{G} \mathcal{N}=0$, so $\left(\mathrm{F}_{i} \mathcal{M}+N\right) /\left(\mathrm{F}_{i-1} \mathcal{M}+N\right) \cong \mathrm{G}_{i} \mathcal{M} / \mathcal{N}=0$ for all 
$i \in \mathbb{Z}$, hence $\mathrm{F}_{i} \mathcal{M} \subseteq \mathrm{F}_{i} \mathcal{M}+N=\mathrm{F}_{i-1} \mathcal{M}+N$ for all $i \in \mathbb{Z}$. In particular $\mathrm{F}_{i_{0}} \mathcal{M} \subseteq \mathrm{F}_{i_{0}-1} \mathcal{M}+N \subseteq N+N=N$, thus $i_{0} \notin I$, a contradiction.

Therefore, under the assumption that $\mathcal{M}$ is discrete, we have the implication $N \subsetneq M \Rightarrow \mathrm{G} \mathcal{N} \subsetneq \mathrm{G} \mathcal{M}$, the property of strict monotony of $\mathrm{G}$ for discrete filtrations.

Remark 1.7. Let $(R, \mathcal{R})$ be a filtered ring. Assume that $\mathcal{R}$ is commutative, that is, $r \in \mathrm{F}_{i} \mathcal{R} \wedge s \in \mathrm{F}_{j} R \Rightarrow r s-s r \in \mathrm{F}_{i+j-1} \mathcal{R}$. Then the ring $\mathrm{G} \mathcal{R}$ is commutative. In this situation let $(I, \mathcal{I})$ be a left ideal of $(R, \mathcal{R})$ and consider the quotient module $(R / I, \mathcal{R} / \mathcal{I})$ of $(R, \mathcal{R})$. Then $\mathrm{G} \mathcal{I}=(0: \mathrm{G} \mathcal{R} / \mathcal{I})$ as ideals of $\mathrm{G} \mathcal{R}$ by Remark 1.5 ,

Definition 1.8. Let $(R, \mathcal{R})$ be a filtered ring and let $(M, \mathcal{M})$ be a left $(R, \mathcal{R})$ module. We define the $\mathcal{M}$-degree function $\operatorname{deg}^{\mathcal{M}}: M \rightarrow \mathbb{Z} \cup\{-\infty\}$ by $\operatorname{deg}^{\mathcal{M}}(m)=$ $\inf \left\{i \in \mathbb{Z} \mid m \in \mathrm{F}_{i} \mathcal{M}\right\}$ for all $m \in M$. In particular, $\operatorname{deg}^{\mathcal{M}}(0)=-\infty$. If $(N, \mathcal{N})$ is a left submodule of $(M, \mathcal{M})$, then $\operatorname{deg}^{\mathcal{N}}(n)=\operatorname{deg}^{\mathcal{M}}(n)$ for all $n \in N$. Further it holds $\operatorname{deg}^{\mathcal{M}}(m+n) \leq \max \left\{\operatorname{deg}^{\mathcal{M}}(m), \operatorname{deg}^{\mathcal{M}}(n)\right\}$ and $\operatorname{deg}^{\mathcal{M}}(r m) \leq \operatorname{deg}^{\mathcal{R}}(r)+\operatorname{deg}^{\mathcal{M}}(m)$ for all $r \in R$ and all $m, n \in M$.

By definiton, we put $\mathrm{F}_{-\infty} \mathcal{M}=0$ and $\mathrm{G}_{-\infty} \mathcal{M}=0$. For each $i \in \mathbb{Z} \cup\{-\infty\}$, let us consider the left $\mathrm{F}_{0} \mathcal{R}$-module epimorphism $\sigma_{i}^{\mathcal{M}}: \mathrm{F}_{i} \mathcal{M} \rightarrow \mathrm{G}_{i} \mathcal{M}$ given by $m \mapsto m+\mathrm{F}_{i-1} \mathcal{M}$. Now we define the $\mathcal{M}$-symbol map $\sigma^{\mathcal{M}}: M \rightarrow \mathrm{G} \mathcal{M}$ of $M$ by $m \mapsto \sigma_{d}^{\mathcal{M}}(m)$ where $d=\operatorname{deg}^{\mathcal{M}}(m)$. We call $\sigma^{\mathcal{M}}(m)$ the $\mathcal{M}$-symbol of $m$. If $(N, \mathcal{N})$ is a left submodule of $(M, \mathcal{M})$, then the image of $\sigma^{\mathcal{N}}(n)$ in GM is precisely $\sigma^{\mathcal{M}}(n)$. Moreover, in general, $\sigma^{\mathcal{M}}$ is not additive, and $\sigma^{\mathcal{M}}$ is multiplicative precisely when $\operatorname{deg}^{\mathcal{M}}(r m)=\operatorname{deg}^{\mathcal{R}}(r)+\operatorname{deg}^{\mathcal{M}}(m)$ for all $r \in R$ and all $m \in M$.

Remark 1.9. Let $(R, \mathcal{R})$ be a filtered $\operatorname{ring},(M, \mathcal{M})$ be a left $(R, \mathcal{R})$-module, and $(N, \mathcal{N})$ be a submodule of $(M, \mathcal{M})$. The image $\sigma^{\mathcal{N}}(N)$ consists precisely of all

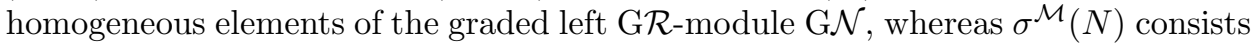

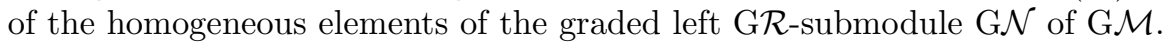

In particular $\mathrm{G} \mathcal{N}$ is generated by $\sigma^{\mathcal{N}}(N)$ as a left $\mathrm{G} \mathcal{R}$-module, and $\mathrm{G} \mathcal{N}$ is

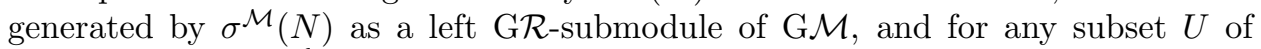

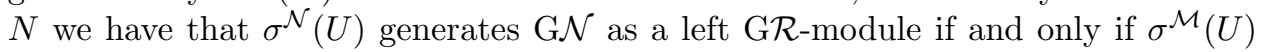
generates $\mathrm{G} \mathcal{N}$ as a left $\mathrm{G} \mathcal{R}$-submodule of GM.

Proposition 1.10. Let $(R, \mathcal{R})$ be a commutatively filtered ring. Let I be a left ideal of $R$ and $\mathcal{I}$ and $\mathcal{R} / \mathcal{I}$ be the induced $\mathcal{R}$-filtrations of $I$ and $R / I$, respectively. Then $(0: \mathrm{GR} / \mathcal{I})=\mathrm{G} \mathcal{I}=\sum_{x \in I} \mathrm{G} \mathcal{R} \sigma^{\mathcal{R}}(x)$ as ideals of $\mathrm{G} \mathcal{R}$.

Proof. This is clear by Remarks 1.7 and 1.9 .

Remark 1.11. Let $(R, \mathcal{R})$ be a filtered $\operatorname{ring}$ and $(M, \mathcal{M})$ be a left $(R, \mathcal{R})$-module. If $U$ is a system of generators of $M$ other than $M$, then GM is not generated by $\sigma^{\mathcal{M}}(U)$, in general.

For instance consider the commutative polynomial ring $R=\mathbb{C}[X]$ provided with the filtration $\mathcal{R}$ given by $\mathrm{F}_{i} \mathcal{R}=\{r \in R \mid \operatorname{deg}(r) \leq i\}$. Put $(M, \mathcal{M})=(R, \mathcal{R})$. Obviously $\{X, X+1\}$ is a system of generators of $M$. Further we have $G \mathcal{R} \cong \mathbb{C}[X]$ as rings and $\mathrm{GM} \cong \mathbb{C}[X]$ as $\mathbb{C}[X]$-modules. In view of these isomorphisms we can write $\sigma^{\mathcal{M}}(X+1)=X=\sigma^{\mathcal{M}}(X)$. Thus $\mathrm{GR} \sigma^{\mathcal{M}}(\{X, X+1\})=\mathbb{C}[X] X \subsetneq \mathbb{C}[X]$.

Remark 1.12. The converse of Remark 1.11 is partially true. If $\mathcal{M}$ is discrete and $U \subseteq M$ is such that $\sigma^{\mathcal{M}}(U)$ generates $\mathrm{G} \mathcal{M}$ over $\mathrm{GR}$, then $U$ generates $M$ over $R$.

Remark 1.13. Let $(R, \mathcal{R})$ be a filtered ring. We can provide the graded ring $G \mathcal{R}$ with its filtration $\mathcal{G R}$ induced by the grading given by $\mathrm{F}_{i} \mathcal{G R}=\bigoplus_{j \leq i} \mathrm{G}_{j} \mathcal{R}$. Then 
we construct the graded ring $G \mathcal{G R}$ associated to the filtered $\operatorname{ring}(\mathrm{G} \mathcal{R}, \mathcal{G R})$. Since for each $i$ one has a left module isomorphism $\mathrm{F}_{i} \mathcal{R} \cong \mathrm{F}_{i} \mathcal{G R}$ over the isomorphic rings $\mathrm{F}_{0} \mathcal{R} \cong \mathrm{F}_{0} \mathcal{G} \mathcal{R}$, there exists a graded ring isomorphism $\mathrm{G} \mathcal{R} \cong \mathrm{GG} \mathcal{R}$.

In a similar manner, if $(M, \mathcal{M})$ is a left $(R, \mathcal{R})$-module, we find an isomorphism $\mathrm{G} \mathcal{M} \cong \mathrm{GG} \mathcal{M}$ of graded left modules over the isomorphic graded rings $\mathrm{G} \mathcal{R} \cong \mathrm{GG \mathcal {R }}$, where $\mathcal{G M}$ is the filtration of GM given by $\mathrm{F}_{i} \mathcal{G M}=\bigoplus_{j \leq i} \mathrm{G}_{j} \mathcal{M}$.

Definition 1.14. Let $(R, \mathcal{R})$ be a filtered ring and $M$ be a left $R$-module. An $\mathcal{R}$ filtration $\mathcal{M}$ of $M$ is good if there exist $s \in \mathbb{N}_{0}, m_{1}, \ldots, m_{s} \in M$, and $p_{1}, \ldots, p_{s} \in \mathbb{Z}$ such that for all $i \in \mathbb{Z}$ it holds $\mathrm{F}_{i} \mathcal{M}=\sum_{j=1}^{s} \mathrm{~F}_{i-p_{j}} \mathcal{R} m_{j}$. Since $1 \in \mathrm{F}_{0} \mathcal{R}$, we then have $m_{j} \in \mathrm{F}_{p_{j}} \mathcal{M}$.

Remark 1.15. In the notation of Definition 1.14, any good $\mathcal{R}$-filtration $\mathcal{M}$ of $M$ is discrete as $\mathcal{R}$ is discrete by definition.

Example 1.16. Let $(R, \mathcal{R})$ be a filtered ring and $M$ be a finitely generated left $R$ module. For each finite system of generators $m \in M^{\oplus s}$ of $M$ and each $p \in \mathbb{Z}^{\oplus s}$ there exists a standard good $\mathcal{R}$-filtration $\mathcal{M}$ of $M$ given by $\mathrm{F}_{i} \mathcal{M}=\sum_{j=1}^{s} \mathrm{~F}_{i-p_{j}} \mathcal{R} m_{j}$.

Proposition 1.17. Let $(R, \mathcal{R})$ be a filtered ring and $(M, \mathcal{M})$ be a left $(R, \mathcal{R})$ module. If the $\mathcal{R}$-filtration $\mathcal{M}$ is good, then the left $\mathrm{GR}$-module $\mathrm{G} \mathcal{M}$ is finitely generated.

Proof. See [13, Lemma I.5.4(2)].

Definition 1.18. Let $(R, \mathcal{R})$ be a filtered $\operatorname{ring},(M, \mathcal{M})$ be a left $(R, \mathcal{R})$-module, and $\left(m_{k}\right)_{k \in \mathbb{N}}$ be a sequence of elements $m_{k}$ of $M$.

Then $\left(m_{k}\right)_{k \in \mathbb{N}}$ is said to be an $\mathcal{M}$-Cauchy sequence if for each $j \in \mathbb{Z}$ there exists $n_{j} \in \mathbb{N}$ such that for all $k, l \geq n_{j}$ it holds $m_{k}-m_{l} \in \mathrm{F}_{j} \mathcal{M}$.

And $\left(m_{k}\right)_{k \in \mathbb{N}}$ is said to be $\mathcal{M}$-convergent to $m \in M$ if for each $j \in \mathbb{Z}$ there exists $n_{j} \in \mathbb{N}$ such that for all $k \geq n_{j}$ it holds $m_{k}-m \in \mathrm{F}_{j} \mathcal{M}$.

If every $\mathcal{M}$-Cauchy sequence of elements of $M$ is $\mathcal{M}$-convergent, then $\mathcal{M}$ is said to be complete.

If $\bigcap_{j \in \mathbb{Z}} \mathrm{F}_{j} \mathcal{M}=\{0\}$, then $\mathcal{M}$ is called separated or Hausdorff.

Remark 1.19. Discrete filtrations are complete and, trivially, separated. So are, in particular, our ring filtrations and any good module filtrations.

Proposition 1.20. Let $(R, \mathcal{R})$ be a filtered ring and $(M, \mathcal{M})$ be a left $(R, \mathcal{R})$ -

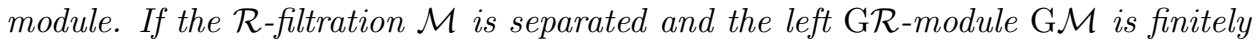
generated, then $\mathcal{M}$ is good.

Proof. As $\mathcal{R}$ is discrete and thus complete, we can appeal to [13, Theorem I.5.7].

Corollary 1.21. Let $(R, \mathcal{R})$ be a filtered ring, $(M, \mathcal{M})$ be a left $(R, \mathcal{R})$-module, and $(N, \mathcal{N})$ be a submodule of $(M, \mathcal{M})$, so that by definition $\mathcal{N}$ is the $\mathcal{R}$-filtration of $N$ induced by $\mathcal{M}$. If the ring $\mathrm{G} \mathcal{R}$ is left noetherian and the $\mathcal{R}$-filtration $\mathcal{M}$ is good, then $\mathcal{N}$ is good, too.

Proof. By Proposition 1.17, GM is left noetherian, and so is GN . By Remark 1.15 $\mathcal{M}$ is discrete, and so is $\mathcal{N}$. We conclude by Remark 1.19 and Proposition 1.20 .

Remark 1.22. Let $(R, \mathcal{R})$ be a filtered ring and $(M, \mathcal{M})$ be a left $(R, \mathcal{M})$-module. Let $N$ be a left $R$-submodule of $M$. If the $\mathcal{R}$-filtration $\mathcal{M}$ is good then the induced $\mathcal{R}$-filtration $\mathcal{M} / \mathcal{N}$ of $M / N$ is good. Indeed, in the notation of Definition 1.14, one immediately sees that $\mathrm{F}_{i} \mathcal{M} / \mathcal{N}=\sum_{j=1}^{s} \mathrm{~F}_{i-p_{j}} \mathcal{R}\left(m_{j}+N\right)$. 
Definition 1.23. Let $(R, \mathcal{R})$ be a filtered ring and $M$ be a left $R$-module. Two $\mathcal{R}$ filtrations $\mathcal{M}^{\prime}$ and $\mathcal{M}^{\prime \prime}$ of $M$ are equivalent or of bounded difference if there exists $r \in \mathbb{N}$, or equivalently $r \in \mathbb{Z}$, such that $\mathrm{F}_{i-r} \mathcal{M}^{\prime \prime} \subseteq \mathrm{F}_{i} \mathcal{M}^{\prime} \subseteq \mathrm{F}_{i+r} \mathcal{M}^{\prime \prime}$ for all $i \in \mathbb{Z}$. Indeed, this defines an equivalence relation among the $\mathcal{R}$-filtrations of $M$.

Proposition 1.24. Let $(R, \mathcal{R})$ be a filtered ring and $\left(M, \mathcal{M}^{\prime}\right)$ and $\left(M, \mathcal{M}^{\prime \prime}\right)$ be left $(R, \mathcal{R})$-modules. If the $\mathcal{R}$-filtrations $\mathcal{M}^{\prime}$ and $\mathcal{M}^{\prime \prime}$ are good, they are equivalent.

Proof. See [13, Lemma I.5.3].

Theorem 1.25. Let $(R, \mathcal{R})$ be a filtered ring such that the ring filtration $\mathcal{R}$ is commutative. Let $\left(M, \mathcal{M}^{\prime}\right)$ and $\left(M, \mathcal{M}^{\prime \prime}\right)$ be left $(R, \mathcal{R})$-modules such that the $\mathcal{R}$ filtrations $\mathcal{M}^{\prime}$ and $\mathcal{M}^{\prime \prime}$ are equivalent. Then $\sqrt{ }\left(0: \mathrm{G} \mathcal{M}^{\prime}\right)=\sqrt{ }\left(0: \mathrm{G} \mathcal{M}^{\prime \prime}\right)$.

Proof. In [13, Lemma III.4.1.9] the claim is stated for good filtrations, but the authors actually prove it for the more general case of equivalent filtrations.

Proposition 1.26. Let $(R, \mathcal{R})$ be a filtered commutative ring, $M$ be an $R$-module, and $N$ be an $R$-submodule of $M$. Providing the annihilators $(0: M),(0: N)$, $(0: M / N)$ in $R$ with the respective induced $\mathcal{R}$-filtrations, denoted $(0: \mathcal{M}),(0: \mathcal{N})$, $(0: \mathcal{M} / \mathcal{N})$, it holds $\sqrt{ } \mathrm{G}(0: \mathcal{M})=\sqrt{ } \mathrm{G}(0: \mathcal{N}) \cap \sqrt{ } \mathrm{G}(0: \mathcal{M} / \mathcal{N})$ in $\mathrm{G} \mathcal{R}$.

Proof. Let $\bar{x} \in \mathrm{G}(0: \mathcal{N}) \cap \mathrm{G}(0: \mathcal{M} / \mathcal{N})$ be a homogeneous element of degree $i \in \mathbb{Z}$. We find $u \in \mathrm{F}_{i}(0: \mathcal{N})=\mathrm{F}_{i} \mathcal{R} \cap(0: N)$ and $v \in \mathrm{F}_{i}(0: \mathcal{M} / \mathcal{N})=\mathrm{F}_{i} \mathcal{R} \cap(0: M / N)$ with $u+\mathrm{F}_{i-1} \mathcal{R}=\bar{x}=v+\mathrm{F}_{i-1} \mathcal{R}$. Because $v \in(0: M / N)$, it holds $v M \subseteq N$. Since $u \in(0: N)$, it follows $u v M=0$. Hence $u v \in(0: M)$. Since $u \in \mathrm{F}_{i} \mathcal{R}$ and $v \in \mathrm{F}_{i} \mathcal{R}$, it follows $u v \in \mathrm{F}_{2 i} \mathcal{R} \cap(0: M)=\mathrm{F}_{2 i}(0: \mathcal{M})$. So $\bar{x}^{2}=u v+\mathrm{F}_{2 i-1} \mathcal{R} \in \mathrm{G}(0: \mathcal{M})$, thus $\bar{x} \in \sqrt{ } \mathrm{G}(0: \mathcal{M})$. We have obtained $\mathrm{G}(0: \mathcal{N}) \cap \mathrm{G}(0: \mathcal{M} / \mathcal{N}) \subseteq \sqrt{ } \mathrm{G}(0: \mathcal{M})$, whereas, on the other hand, as $(0: M) \subseteq(0: N) \cap(0: M / N)$, it follows from 1.6 that $\mathrm{G}(0: \mathcal{M}) \subseteq \mathrm{G}(0: \mathcal{N}) \cap \mathrm{G}(0: \mathcal{M} / \mathcal{N})$. Now we pass to the radicals.

Remark 1.27. Let $(R, \mathcal{R})$ be a filtered ring and $\phi: M \rightarrow N$ be an isomorphism of left $R$-modules. If $\mathcal{M}$ is an $\mathcal{R}$-filtration of $M$, then there exists an $\mathcal{R}$-filtration $\mathcal{N}$ of $N$ induced by $\phi$ given by $\mathrm{F}_{i} \mathcal{N}=\phi\left(\mathrm{F}_{i} \mathcal{M}\right)$ such that there exists a graded GR -isomorphism G $\phi: \mathrm{G} \mathcal{M} \rightarrow \mathrm{G} \mathcal{N}$ induced by $\phi$; see Remark 1.5. Moreover, if $\mathcal{M}$ is good, then $\mathcal{N}$ is good, as one easily checks.

Proposition 1.28. Let $R$ be a commutative ring and $\mathcal{R}$ be a filtration of $R$ such that induced $\mathcal{R}$-filtrations on submodules and quotient modules of $R$ are good. Let $M$ be a finitely generated $R$-module and $\mathcal{M}$ be an $\mathcal{R}$-filtration such that induced $\mathcal{R}$-filtrations on submodules and quotient modules of $M$ are good. Consider the annihilator $(0: M)$ of $M$ in $R$ provided with its induced $\mathcal{R}$-filtration, which we denote by $(0: \mathcal{M})$. Then $\sqrt{ } \mathrm{G}(0: \mathcal{M})=\sqrt{ }(0: \mathrm{G} \mathcal{M})$ as ideals of the commutative ring $\mathrm{G} \mathcal{R}$.

Proof. We find $t \in \mathbb{N}$ such that $M$ is generated by $t$ elements. If $t=1$, there exists an $R$-module isomorphism $\phi: M \rightarrow R / I$ for some ideal $I$ of $R$. We furnish the $R$ module $R / I$ with the induced $\mathcal{R}$-filtration $\mathcal{R} / \mathcal{I}$, good by hypothesis, and with the $\phi$ induced $\mathcal{R}$-filtration, denoted $\phi(\mathcal{M})$, which is good by Remark 1.27 since $\mathcal{M}$ is good by hypothesis. By Remark 1.27 $(0: \mathrm{G} \mathcal{M})=(0: \mathrm{G} \phi(\mathcal{M}))$. By Proposition 1.24 and Theorem 1.25, $\sqrt{ }(0: \mathrm{G} \phi(\mathcal{M}))=\sqrt{ }(0: \mathrm{GR} / \mathcal{I})$. As $(0: M)=(0: R / I)=I$, $(0: \mathcal{M})$ is precisely the induced $\mathcal{R}$-filtration of $I$, hence by Remark 1.7 we have $(0: \mathrm{G} \mathcal{R} / \mathcal{I})=\mathrm{G}(0: \mathcal{M})$. Thus $\sqrt{ }(0: \mathrm{G} \mathcal{M})=\sqrt{ } \mathrm{G}(0: \mathcal{M})$. 
Now let $t>1$. Assume inductively that the statement holds for all $R$-modules generated by less than $t$ elements. We find a cyclic submodule $N$ of $M$ such that $M / N$ is generated over $R$ by $t-1$ elements. We provide $N$ and $M / N$ by the respective induced filtrations $\mathcal{N}$ and $\mathcal{M} / \mathcal{N}$, which are good, and we provide the ideals $(0: N)$ and $(0: M / N)$ of $R$ by the respective induced filtrations, denoted $(0: \mathcal{N})$ and $(0: \mathcal{M} / \mathcal{N})$, which are good by hypothesis. By the case with $t=1$, we have $\sqrt{ } \mathrm{G}(0: \mathcal{N})=\sqrt{ }(0: \mathrm{G} \mathcal{N})$. By the induction hypothesis, we have $\sqrt{ } \mathrm{G}(0: \mathcal{M} / \mathcal{N})=$ $\sqrt{ }(0: \mathrm{G} \mathcal{M} / \mathcal{N})$. The short exact sequence $N \longmapsto M \rightarrow M / N$ of filtered $R$-modules induces the short exact sequence $\mathrm{G} \mathcal{N} \longmapsto \mathrm{G} \mathcal{M} \rightarrow \mathrm{G} \mathcal{M} / \mathcal{N}$ of graded $\mathrm{G} \mathcal{R}$-modules; see Remark 1.5. Therefore $\sqrt{ }(0: \mathrm{G} \mathcal{M})=\sqrt{ }(0: \mathrm{G} \mathcal{N}) \cap \sqrt{ }(0: \mathrm{G} \mathcal{M} / \mathcal{N})$, and hence $\sqrt{ }(0: \mathrm{G} \mathcal{M})=\sqrt{ } \mathrm{G}(0: \mathcal{N}) \cap \sqrt{ } \mathrm{G}(0: \mathcal{M} / \mathcal{N})$. By Proposition 1.26] we finally get $\sqrt{ }(0: \mathrm{G} \mathcal{M})=\sqrt{ } \mathrm{G}(0: \mathcal{M})$.

Remark 1.29. We finish this section with a remark that will be useful later on. Let $R$ be a commutative ring and $\mathcal{R}$ be a filtration of $R$, so that $\mathcal{R}$ trivially is commutative. Let $I$ be an ideal of $R$ and provide $I$ with its induced $\mathcal{R}$-filtration, denoted $\mathcal{I}$, and provide $\sqrt{ } I$ with its induced $\mathcal{R}$-filtration, denoted $\sqrt{ } \mathcal{I}$. Then $\sqrt{ } \mathrm{G} \sqrt{ } \mathcal{I}=\sqrt{ } \mathrm{G} \mathcal{I}$. Indeed let $\bar{x} \in \mathrm{G} \sqrt{ } \mathcal{I}$ be a homogeneous element of degree $i \in \mathbb{Z}$. So $\bar{x}=x+\mathrm{F}_{i-1} \mathcal{R}$ for some $x \in \mathrm{F}_{i} \sqrt{ } \mathcal{I}=\mathrm{F}_{i} \mathcal{R} \cap \sqrt{ } I$. We find $k \in \mathbb{N}$ such that $x^{k} \in I$, and so $x^{k} \in \mathrm{F}_{k i} \mathcal{R} \cap I=\mathrm{F}_{k i} \mathcal{I}$, thus $\bar{x}^{k}=x^{k}+\mathrm{F}_{k i-1} \mathcal{R} \in \mathrm{G} \mathcal{I}$, hence $\bar{x} \in \sqrt{ } \mathrm{G} \mathcal{I}$. We have shown that $\mathrm{G} \sqrt{ } \mathcal{I} \subseteq \sqrt{ } \mathrm{G} \mathcal{I}$. On the other hand, by Remark1.6, we have $\mathrm{G} \mathcal{I} \subseteq \mathrm{G} \sqrt{ } \mathcal{I}$. Passing to the radicals, the claim follows.

\section{WEYL ALGEBRAS}

In this section let $n \in \mathbb{N}$ and $K$ be a field of characteristic 0 . We write $K[X, Y]$ for the commutative polynomial ring $K\left[X_{1}, \ldots, X_{n}, Y_{1}, \ldots, Y_{n}\right]$ and denote its subring $K\left[X_{1}, \ldots, X_{n}\right]$ by $K[X]$.

For all $(r, s) \in \mathbb{N}_{0} \times \mathbb{N}_{0}$, we write $(r \mid s)$ for the vector $\omega \in \mathbb{N}_{0}^{2 n}$ with $\omega_{i}=r$ and $\omega_{n+i}=s$ for $1 \leq i \leq n$. For all $\alpha, \beta \in \mathbb{N}_{0}^{n}$, we write $(\alpha \mid \beta)$ for the vector $\omega \in \mathbb{N}_{0}^{2 n}$ with $\omega_{i}=\alpha_{i}$ and $\omega_{n+i}=\beta_{i}$ for $1 \leq i \leq n$. For all $t \in \mathbb{N}$ and all $\alpha, \beta \in \mathbb{N}_{0}^{t}$, we denote the sum $\sum_{i=1}^{t} \alpha_{i} \beta_{i}$ by $\alpha \cdot \beta$. For all $i \in\{1, \ldots, n\}$, we put $\varepsilon^{i}=\left(\delta_{i j}\right)_{j=1}^{n} \in \mathbb{N}_{0}^{n}$, where $\delta_{i j} \in \mathbb{N}_{0}$ is the Kronecker symbol.

We introduce Weyl algebras over $K$ and state some facts about them. In doing this, we generalize certain well-known results that are proved for instance in [8]; here the missing proofs of Proposition 2.4 and Theorem 2.9 are elementary but tedious computations and can be mimicked word for word from [8].

Definition 2.1. The $n^{\text {th }}$ Weyl algebra $W$ over $K$ is defined as the $K$-subalgebra $K\left\langle\xi_{1}, \ldots, \xi_{n}, \partial_{1}, \ldots, \partial_{n}\right\rangle$ of $\operatorname{End}_{K}(K[X])$ generated by the $K$-linear endomorphisms $\xi_{1}, \ldots, \xi_{n}$ and $\partial_{1}, \ldots, \partial_{n}$ of $K[X]$ given by $\xi_{i}(p)=X_{i} p$ and $\partial_{i}(p)=\frac{\partial p}{\partial X_{i}}$ for all $p \in K[X]$. The generators satisfy the Heisenberg commutation rules:

(a) $\left[\xi_{i}, \xi_{j}\right]=0$,

(b) $\left[\partial_{i}, \partial_{j}\right]=0$,

(c) $\left[\xi_{i}, \partial_{j}\right]+\delta_{i j}=0$,

where $\delta_{i j} \in K$ is the Kronecker symbol.

Remark 2.2. As a $K$-module, $W$ has a canonical basis $\left\{\xi^{\lambda} \partial^{\mu} \mid(\lambda, \mu) \in \mathbb{N}_{0}^{n} \times \mathbb{N}_{0}^{n}\right\}$; see [7. Satz 2.7] or [8, Proposition 1.2.1]. As a consequence, for each $w \in W$ there exists a unique function $c_{w}: \mathbb{N}_{0}^{n} \times \mathbb{N}_{0}^{n} \rightarrow K$ of finite support $\operatorname{supp}(w)=$ $\left\{(\lambda, \mu) \in \mathbb{N}_{0}^{n} \times \mathbb{N}_{0}^{n} \mid c_{w}(\lambda, \mu) \neq 0\right\}$ such that $w=\sum c_{w}(\lambda, \mu) \xi^{\lambda} \partial^{\mu}$ with the sum 
taken over all $(\lambda, \mu) \in \operatorname{supp}(w)$. We write $c_{\lambda \mu}$ for $c_{w}(\lambda, \mu)$ and say that $\sum c_{\lambda \mu} \xi^{\lambda} \partial^{\mu}$ is the canonical form of $w$.

Definition 2.3. Let $\operatorname{deg}^{\omega}(w)=\sup \{\omega \cdot(\lambda \mid \mu) \mid(\lambda, \mu) \in \operatorname{supp}(w)\}$ for all $\omega \in \mathbb{N}_{0}^{2 n}$ and all $w \in W$, the $\omega$-degree of $w$ with values in $\mathbb{Z} \cup\{-\infty\}$.

Proposition 2.4. Let $\omega \in \mathbb{N}_{0}^{2 n}$ and let $u, v \in W$. Then one has

(a) $\operatorname{deg}^{\omega}(u+v) \leq \max \left\{\operatorname{deg}^{\omega}(u), \operatorname{deg}^{\omega}(u)\right\}$,

(b) $\operatorname{deg}^{\omega}([u, v]) \leq \operatorname{deg}^{\omega}(u)+\operatorname{deg}^{\omega}(v)-\min _{1 \leq i \leq n}\left\{\omega_{i}+\omega_{n+i}\right\}$,

(c) $\operatorname{deg}^{\omega}(u v)=\operatorname{deg}^{\omega}(u)+\operatorname{deg}^{\omega}(v)$.

Equality holds in (a) if $\operatorname{deg}^{\omega}(u) \neq \operatorname{deg}^{\omega}(v)$.

Definition 2.5. Let $\omega \in \mathbb{N}_{0}^{2 n}$. Consider the family $\mathrm{F}^{\omega} W=\left(\mathrm{F}_{i}^{\omega} W\right)_{i \in \mathbb{Z}}$ defined by $\mathrm{F}_{i}^{\omega} W=\left\{w \in W \mid \operatorname{deg}^{\omega}(w) \leq i\right\}$. Then $\mathrm{F}^{\omega} W$ is a filtration of $W$ by Proposition 2.4. We denote by $\mathrm{G}^{\omega} W$ the associated graded ring of $W$ with respect to $\mathrm{F}^{\omega} W$, and by $\mathrm{G}_{i}^{\omega} W$ the $i^{\text {th }}$ homogeneous component of $\mathrm{G}^{\omega} W$.

Given any $\mathrm{F}^{\omega} W$-filtration $\mathrm{F}^{\omega} M=\left(\mathrm{F}_{i}^{\omega} M\right)_{i \in \mathbb{Z}}$ of a left $W$-module $M$, we denote by $\mathrm{G}^{\omega} M$ the associated graded left $\mathrm{G}^{\omega} W$-module associated to $M$ with respect to $\mathrm{F}^{\omega} M$, and by $\mathrm{G}_{i}^{\omega} M$ the $i^{\text {th }}$ homogeneous component of $\mathrm{G}^{\omega} M$.

We write $\sigma^{\omega}$ for the symbol map $W \rightarrow \mathrm{G}^{\omega} W$, and $\sigma_{i}^{\omega}$ for the $i^{\text {th }}$ symbol map $\mathrm{F}_{i}^{\omega} W \rightarrow \mathrm{G}_{i}^{\omega} W$. Thus $\sigma^{\omega}(w)=\sigma_{\operatorname{deg}^{\omega}(w)}^{\omega}(w)$ for all $w \in W$.

Definition 2.6. We define $\Omega=\left\{\omega \in \mathbb{N}_{0}^{2 n} \mid \omega_{i}+\omega_{n+i}>0\right.$ whenever $\left.1 \leq i \leq n\right\}$, the natural polynomial region of $W$.

Remark 2.7. Let $\omega \in \Omega$ and $v, w \in W$. As $\operatorname{deg}^{\omega}(u v)=\operatorname{deg}^{\omega}(u)+\operatorname{deg}^{\omega}(v)$ by Proposition 2.4, it holds $\sigma^{\omega}(u v)=\sigma^{\omega}(u) \sigma^{\omega}(v)$.

Remark 2.8. For all $\omega \in \Omega$, the filtration $\mathrm{F}^{\omega} W$ of $W$ is commutative by Proposition 2.4. so that the ring $\mathrm{G}^{\omega} W$ is commutative.

Remarks 2.7 and 2.8, the canonical injection $K \longmapsto \mathrm{G}^{\omega} W$, and the universal property of commutative polynomial rings imply the following theorem.

Theorem 2.9. For each $\omega \in \Omega$, there exists an isomorphism of commutative $K$ algebras $\psi^{\omega}: K[X, Y] \rightarrow \mathrm{G}^{\omega} W$ given by

$$
\sum_{(\lambda, \mu) \in \mathbb{N}_{0}^{n} \times \mathbb{N}_{0}^{n}} c_{\lambda \mu} X^{\lambda} Y^{\mu} \mapsto \sum_{(\lambda, \mu) \in \mathbb{N}_{0}^{n} \times \mathbb{N}_{0}^{n}} c_{\lambda \mu} \sigma^{\omega}\left(\xi^{\lambda}\right) \sigma^{\omega}\left(\partial^{\mu}\right),
$$

which is graded if we put $\operatorname{deg}\left(X_{i}\right)=\omega_{i}$ and $\operatorname{deg}\left(Y_{i}\right)=\omega_{n+i}$ for all $1 \leq i \leq n$.

Remark 2.10. By Theorem[2.9, Remark[1.12, and Proposition 2.4, the Weyl algebras are left noetherian domains.

Remark 2.11. All of what we have defined and said in this section about Weyl algebras can be done and proved in the same way for the commutative polynomial ring $K[X, Y]$ also. In this situation we may even drop the hypothesis that the field be of characteristic 0 and may consider whole $\mathbb{N}_{0}^{2 n}$ instead of $\Omega$. We shall use a similar notation as introduced above for Weyl algebras, with one exception: given any $\nu \in \mathbb{N}_{0}^{2 n}$, we shall write $\tau_{i}^{\nu}$ for the $i^{\text {th }}$ symbol map $\mathrm{F}_{i}^{\nu} K[X, Y] \rightarrow \mathrm{G}_{i}^{\nu} K[X, Y]$ and $\tau^{\nu}$ for the symbol map $K[X, Y] \rightarrow \mathrm{G}^{\nu} K[X, Y]$, in order to distinguish them from the symbol maps of the $n^{\text {th }}$ Weyl algebra. 


\section{GRÖBNER BASES IN WEYL ALGEBRAS}

In this section we recall the notion of universal Gröbner bases in Weyl algebras and state their existence. The proof of this fact can be found in [5] see also [17]. In [16] the same statement is proved for commutative polynomial rings; a similar proof exists for Weyl algebras.

We keep the notation of the previous section, and denote by $M$ the canonical $K$-basis $\left\{X^{\lambda} Y^{\mu} \mid(\lambda, \mu) \in \mathbb{N}_{0}^{n} \times \mathbb{N}_{0}^{n}\right\}$ of $K[X, Y]$ consisting of the monomials $X^{\lambda} Y^{\mu}$, and by $N$ the canonical $K$-basis $\left\{\xi^{\lambda} \partial^{\mu} \mid(\lambda, \mu) \in \mathbb{N}_{0}^{n} \times \mathbb{N}_{0}^{n}\right\}$ of $W$ consisting of the normal monomials $\xi^{\lambda} \partial^{\mu}$.

For each $\omega \in \Omega$ we shall tacitly identify the $\operatorname{ring} \mathrm{G}^{\omega} W$ with $K[X, Y]$ by means of the $K$-algebra isomorphism $\psi^{\omega}$ of Theorem 2.9 and hence for each left ideal $L$ consider $\mathrm{G}^{\omega} L$ as an ideal of $K[X, Y]$. Similarly for each $\nu \in \mathbb{N}_{0}^{2 n}$, we shall identify $\mathrm{G}^{\nu} K[X, Y]$ with $K[X, Y]$ and thus for each ideal $I$ of $K[X, Y]$ consider $\mathrm{G}^{\nu} I$ as an ideal of $K[X, Y]$.

Definition 3.1. A normal ordering, or monomial ordering in 9, or admissible ordering in [17, or term ordering in [15], is a total ordering $\preceq$ on $\mathbb{N}_{0}^{n} \times \mathbb{N}_{0}^{n}$ such that it holds:

(a) well-foundedness, $(0,0) \preceq(\lambda, \mu)$, and

(b) compatibility, $(\lambda, \mu) \preceq(\rho, \sigma) \Rightarrow(\lambda+\alpha, \mu+\beta) \preceq(\rho+\alpha, \sigma+\beta)$.

With abuse of notation we write $\xi^{\lambda} \partial^{\mu} \preceq \xi^{\rho} \partial^{\sigma}$ and $X^{\lambda} Y^{\mu} \preceq X^{\rho} Y^{\sigma}$ whenever $(\lambda, \mu) \preceq(\rho, \sigma)$. We denote by $\mathcal{O}$ the set of all normal orderings.

Example 3.2. Lexicographic orderings are normal orderings.

Remark 3.3. There exists a $K$-module isomorphism $\Phi: W \rightarrow K[X, Y]$ which maps the canonical basis $N$ of $W$ to the canonical basis $M$ of $K[X, Y]$ by the assignment $\xi^{\lambda} \partial^{\mu} \mapsto X^{\lambda} Y^{\mu}$.

Notation 3.4. Let $\preceq \in \mathcal{O}$. For $w \in W \backslash\{0\}$ we write $\operatorname{lm}_{\preceq}(w)$ for the greatest normal monomial in the canonical form of $w$ with respect to $\preceq$. We denote $\Phi\left(\operatorname{lm}_{\preceq}(w)\right)$ by $\mathrm{LM}_{\preceq}(w)$. Given $L \subseteq W$, we often denote by $\operatorname{LM}_{\preceq}(L)$ the ideal $\sum_{x \in L \backslash\{0\}} K[X, Y] \bar{L}_{\preceq}(x)$ of $K[X, Y]$. For $p \in K[X, Y] \backslash\{0\}$ and $I \subseteq K[X, Y]$, we define $\mathrm{LM}_{\preceq}(p)$ and $\mathrm{LM}_{\preceq}(I)$ similarly.

Definition 3.5. Let $L \subseteq W$ be a left ideal, and let $\preceq \in \mathcal{O}$. According to [15, we say that a finite subset $B$ of $L$ is a Gröbner basis of $L$ with respect to $\preceq$, or a $\preceq$-Gröbner basis of $L$, if it holds $L=\sum_{b \in B} W b$ and $\operatorname{LM}_{\preceq}(L)=\sum_{b \in B \backslash\{0\}} K[X, Y] \mathrm{LM}_{\preceq}(b)$. Similarly we define a $\preceq$-Gröbner basis of an ideal $I \subseteq K[X, Y]$; see [9].

Theorem 3.6. Let $L \subseteq W$ be a left ideal and let $\preceq \in \mathcal{O}$. Then $L$ admits a Gröbner basis with respect to $\preceq$.

Proof. See [5, Corollary 1.9.7] or [5, Theorem 2.3.2] or [15, Theorem 1.1.10].

Definition 3.7. Let $L$ be a left ideal of $W$. A finite subset $U$ of $L$ is a universal Gröbner basis of $L$ if $U$ is a $\preceq$-Gröbner basis of $L$ for each normal ordering $\preceq$.

Theorem 3.8. Each left ideal $L$ of $W$ admits a universal Gröbner basis.

Proof. See [5, Corollary 1.10.5 and Example 1.8.2] or [5, Theorem A.2.4].

Remark 3.9. For each $\nu \in \mathbb{N}_{0}^{2 n}$ and each $\preceq \in \mathcal{O}$, there exists $\preceq_{\nu} \in \mathcal{O}$ defined by $\xi^{\lambda} \partial^{\mu} \preceq_{\nu} \xi^{\rho} \partial^{\sigma} \Leftrightarrow(\lambda \mid \mu) \cdot \nu<(\rho \mid \sigma) \cdot \nu \vee((\lambda \mid \mu) \cdot \nu=(\rho \mid \sigma) \cdot \nu \wedge(\lambda, \mu) \preceq(\rho, \sigma))$. 
Theorem 3.10. Let $\omega \in \Omega, \preceq \in \mathcal{O}, L \subseteq W$ be a left ideal, and $B$ be $a \preceq_{\omega}$-Gröbner basis of $L$. Then $\sigma^{\omega}(B)$ is a $\preceq$-Gröbner basis of $\mathrm{G}^{\omega} L$, thus $\mathrm{G}^{\omega} L=\left\langle\sigma^{\omega}(b) \mid b \in B\right\rangle$ and $\mathrm{LM}_{\preceq}\left(\mathrm{G}^{\omega} L\right)=\left\langle\mathrm{LM}_{\preceq}\left(\sigma^{\omega}(b)\right) \mid b \in B\right\rangle$ as ideals of $K[X, Y]$.

Proof. See [15, Theorem 1.1.6(1)] or [12, Propositions V.7.2 and II.4.2] or [5, Theorem 2.3.8].

Remark 3.11. Analogously as in Theorem 3.10, if $\nu \in \mathbb{N}_{0}^{2 n}, \preceq \in \mathcal{O}, I \subseteq K[X, Y]$ is an ideal, $B$ is a ${ }_{\nu}$-Gröbner basis of $I$, then $\tau^{\nu}(B)$ is a $\preceq$-Gröbner basis of $\mathrm{G}^{\nu} I$.

Corollary 3.12. For every left ideal $L$ of $W$ the set $\left\{\mathrm{G}^{\omega} L \mid \omega \in \Omega\right\}$ is finite. Similarly, for every ideal I of $K[X, Y]$ the set $\left\{\mathrm{G}^{\nu} I \mid \nu \in \mathbb{N}_{0}^{2 n}\right\}$ is finite.

Proof. By Theorem 3.8, we can find a universal Gröbner basis $U \supseteq\{0\}$ of $L$. By Theorem 3.10, $\mathrm{G}^{\omega} L=\left\langle\sigma^{\omega}(u) \mid u \in U\right\rangle$. It follows that $\#\left\{\mathrm{G}^{\omega} L \mid \omega \in \Omega\right\} \leq$ $\prod_{u \in U} \sum_{0 \leq k \leq \# \operatorname{supp}(u)}\left(\begin{array}{c}\# \operatorname{supp}(u) \\ k\end{array}\right)<\infty$.

Remark 3.13. Another proof of Corollary 3.12 by homogenization can be found in [2, Theorem 3.6].

\section{Characteristic varieties over Weyl algebras}

We encounter the notion of characteristic variety and critical cone and prove our main result, from which a relation between characteristic varieties and critical cones follows. We keep the notation of the previous section.

Remark 4.1. Fix any $\omega \in \Omega$. By Theorem 2.9. $\mathrm{G}^{\omega} W \cong K[X, Y]$ as $K$-algebras. Let $M$ be finitely generated left $W$-module. By Example 1.16 we can provide $M$ with a good $\omega$-filtration $\mathrm{F}^{\omega} M$. By Proposition 1.17 the $K[X, Y]$-module $\mathrm{G}^{\omega} M$ is finitely generated, and by Remark 2.8. Proposition [1.24, and Theorem 1.25 the ideal $\sqrt{ }\left(0: \mathrm{G}^{\omega} M\right)$ of $K[X, Y]$ is independent of the choice of $\mathrm{F}^{\omega} M$.

Definition 4.2. Let $\omega \in \Omega$ and let $M$ be a finitely generated left $W$-module. By Remark 4.1 we may define the $\omega$-characteristic variety $\mathcal{V}^{\omega}(M)$ of $M$ as the closed set $\operatorname{Var}\left(\sqrt{ }\left(0: \mathrm{G}^{\omega} M\right)\right)=\operatorname{Var}\left(0: \mathrm{G}^{\omega} M\right)$ of $\operatorname{Spec}(K[X, Y])$. In particular we consider $\mathcal{V}^{(1 \mid 1)}(M)$ and $\mathcal{V}^{(0 \mid 1)}(M)$, the characteristic variety of $M$ by degree and by order.

We define the $\omega$-critical cone $\mathcal{C}^{\omega}(M)$ of $M$ as $\operatorname{Var}\left(\mathrm{G}^{(1 \mid 1)} \sqrt{ }\left(0: \mathrm{G}^{\omega} M\right)\right)$, which is equal to $\operatorname{Var}\left(\mathrm{G}^{(1 \mid 1)}\left(0: \mathrm{G}^{\omega} M\right)\right)$ and $\operatorname{Var}\left(0: \mathrm{G}^{(1 \mid 1)} \mathrm{G}^{\omega} M\right)$ by Remark 1.29 and Proposition 1.28 a closed set of $\operatorname{Spec}(K[X, Y])$. In particular we consider $\mathcal{C}^{(1 \mid 1)}(M)$ and $\mathcal{C}^{(0 \mid 1)}(M)$, the critical cone of $M$ by degree and by order.

Remark 4.3. Let $M$ be a finitely generated left $W$-module and $N$ be a submodule of $M$. If $M$ is provided with a good filtration, by Theorem 2.9 and by Corollary 1.21 and Remark 1.22 the induced $\omega$-filtrations of $N$ and $M / N$ are good. Therefore what is said in Remark 4.1 and Definition 4.2 applies also to $N$ and $M / N$.

Theorem 4.4. Given any finitely generated left $W$-module $M$, there are only finitely many distinct characteristic varieties $\mathcal{V}^{\omega}(M)$ for $\omega$ varying in $\Omega$.

Proof. If $N \subseteq M$ is a submodule by Remark 1.5 one has $\mathcal{V}^{\omega}(M)=\mathcal{V}^{\omega}(N) \cup \mathcal{V}^{\omega}(M / N)$ for all $\omega \in \Omega$. By induction over the number of generators of $M$, the claim follows from Corollary 3.12 and Remark 1.7 . 
Lemma 4.5. Let $w \in W, \nu \in \mathbb{N}_{0}^{2 n}, \omega \in \Omega$. Let $l \in \mathbb{N}_{0}$ with $l \geq \operatorname{deg}^{\nu}(w)$ in $W$, let $m \in \mathbb{N}_{0}$ with $m \geq \operatorname{deg}^{\omega}(w)$ in $W$, and let $p \in \mathbb{N}_{0}$ with $p \geq \operatorname{deg}^{\nu}\left(\sigma_{m}^{\omega}(w)\right)$ in $K[X, Y]$. Then in $K[X, Y]$ for all $s \in \mathbb{N}$ such that $s>l-p$ it holds $\tau_{p}^{\nu}\left(\sigma_{m}^{\omega}(w)\right)=\sigma_{p+s m}^{\nu+s \omega}(w)$.

Proof. We write $w$ in canonical form as $\sum_{(\lambda, \mu) \in \mathbb{S}} c_{\lambda \mu} \xi^{\lambda} \partial^{\mu}$, where $\mathbb{S}=\operatorname{supp}(w)$ and $c_{\lambda \mu} \in K \backslash\{0\}$. By definition, we have $\omega \cdot(\lambda \mid \mu) \leq m$ for all $(\lambda, \mu) \in \mathbb{S}$. Hence $\sigma_{m}^{\omega}(w)=\sum_{(\lambda, \mu) \in \mathbb{S}_{m}} c_{\lambda \mu} X^{\lambda} Y^{\mu}$, where $\mathbb{S}_{m}=\{(\lambda, \mu) \in \mathbb{S} \mid \omega \cdot(\lambda \mid \mu)=m\}$. Similarly, $\nu \cdot(\lambda \mid \mu) \leq p$ for all $(\lambda, \mu) \in \mathbb{S}_{m}$. Hence $\tau_{p}^{\nu}\left(\sigma_{m}^{\omega}(w)\right)=\sum_{(\lambda, \mu) \in \mathbb{S}_{m, p}} c_{\lambda \mu} X^{\lambda} Y^{\mu}$, where $\mathbb{S}_{m, p}=\left\{(\lambda, \mu) \in \mathbb{S}_{m} \mid \nu \cdot(\lambda \mid \mu)=p\right\}$.

Let $(\lambda, \mu) \in \mathbb{S}$. As just observed, $\omega \cdot(\lambda \mid \mu) \leq m$, and moreover if $\omega \cdot(\lambda \mid \mu)=m$, then $\nu \cdot(\lambda \mid \mu) \leq p$. Thus we have the following three cases.

If $\omega \cdot(\lambda \mid \mu)=m$ and $\nu \cdot(\lambda \mid \mu)=p$, then $(\nu+s \omega) \cdot(\lambda \mid \mu)=\nu \cdot(\lambda \mid \mu)+s \omega \cdot(\lambda \mid \mu)=$ $p+s m$, hence $\xi^{\lambda} \partial^{\mu} \in \mathrm{F}_{p+s m}^{\nu+s \omega} W \backslash \mathrm{F}_{p+s m-1}^{\nu+s \omega} W$ for all $s \in \mathbb{N}$.

If $\omega \cdot(\lambda \mid \mu)=m$ and $\nu \cdot(\lambda \mid \mu)<p$, then $(\nu+s \omega) \cdot(\lambda \mid \mu)=\nu \cdot(\lambda \mid \mu)+s \omega \cdot(\lambda \mid \mu)<$ $p+s m$, hence $\xi^{\lambda} \partial^{\mu} \in \mathrm{F}_{p+s m-1}^{\nu+s \omega} W$ for all $s \in \mathbb{N}$.

If $\omega \cdot(\lambda \mid \mu)<m$, then $(\nu+s \omega) \cdot(\lambda \mid \mu)=\nu \cdot(\lambda \mid \mu)+s \omega \cdot(\lambda \mid \mu) \leq l+s m-s<p+s m$ as soon as $s>l-p$, hence $\xi^{\lambda} \partial^{\mu} \in \mathrm{F}_{p+s m-1}^{\nu+s \omega} W$ for all $s \in \mathbb{N}$ with $s>l-p$.

Therefore, putting $\mathbb{S}_{m, p}^{\prime}=\{(\lambda, \mu) \in \mathbb{S} \mid \omega \cdot(\lambda \mid \mu)=m, \nu \cdot(\lambda \mid \mu)=p\}$, we obtain $\sigma_{p+s m}^{\nu+s \omega}(w)=\sum_{(\lambda, \mu) \in \mathbb{S}_{m, p}^{\prime}} c_{\lambda \mu} X^{\lambda} Y^{\mu}$ for all $s \in \mathbb{N}$ with $s>l-p$. Since $\mathbb{S}_{m, p}=\mathbb{S}_{m, p}^{\prime}$, we are done.

Lemma 4.6. Let $w \in W$, and let $\nu \in \mathbb{N}_{0}^{2 n}$ and $\omega \in \Omega$. Then for all $s \in \mathbb{N}$ such that $s>\operatorname{deg}^{\nu}(w)-\operatorname{deg}^{\nu}\left(\sigma^{\omega}(w)\right)$ it holds $\operatorname{deg}^{\nu}\left(\sigma^{\omega}(w)\right)+s \operatorname{deg}^{\omega}(w)=\operatorname{deg}^{\nu+s \omega}(w)$.

Proof. If $w=0$, then the statement holds for all $s \in \mathbb{N}$. Hence let $w \neq 0$, and put $l=\operatorname{deg}^{\nu}(w), m=\operatorname{deg}^{\omega}(w)$, and $p=\operatorname{deg}^{\nu}\left(\sigma_{m}^{\omega}(w)\right)$. Let $s \in \mathbb{N}$ with $s>l-p$ and put $d=\operatorname{deg}^{\nu+s \omega}(w)$. As in the proof of Lemma4.5 we obtain $(\nu+s \omega) \cdot(\lambda \mid \mu) \leq p+s m$ for all $(\lambda, \mu) \in \operatorname{supp}(w)$, hence $d=\sup \{(\nu+s w) \cdot(\lambda \mid \mu) \mid(\lambda, \mu) \in \operatorname{supp}(w)\} \leq$ $p+s m$. If it held that $d<p+s m$, then we would have $\sigma_{p+s m}^{\nu+s \omega}(w)=0$, whereas $\tau_{p}^{\nu}\left(\sigma_{m}^{\omega}(w)\right) \neq 0$, in contradiction to Lemma 4.5. Hence $p+s m=d$, our claim.

Lemma 4.7. Let $w \in W$, and let $\nu \in \mathbb{N}_{0}^{2 n}$ and $\omega \in \Omega$. Then for all $s \in \mathbb{N}$ such that $s>\operatorname{deg}^{\nu}(w)-\operatorname{deg}^{\nu}\left(\sigma^{\omega}(w)\right)$, it holds $\tau^{\nu}\left(\sigma^{\omega}(w)\right)=\sigma^{\nu+s \omega}(w)$.

Proof. This is proven by Lemma 4.5 with $l=\operatorname{deg}^{\nu}(w), m=\operatorname{deg}^{\omega}(w)$, and $p=$ $\operatorname{deg}^{\nu}\left(\sigma_{m}^{\omega}(w)\right)=\operatorname{deg}^{\nu}\left(\sigma^{\omega}(w)\right)$, and by Lemma 4.6

Theorem 4.8 extends a result published in 1971 by Bernstein as a part of the proof of [4, Theorem 3.1], namely that $\mathrm{G}^{(1 \mid 1)} \mathrm{G}^{(0 \mid 1)} L \subseteq \mathrm{G}^{(1 \mid s)} L$ for $s \gg 0$. In greater generality we prove the converse inclusion also.

Theorem 4.8. Let $L$ be a left ideal of $W$. For all $\nu \in \mathbb{N}_{0}^{2 n}$ there exists $s_{\nu} \in \mathbb{N}_{0}$ such that for all $\omega \in \Omega$ and all $s \in \mathbb{N}$ with $s>s_{\nu}$, it holds that $\mathrm{G}^{\nu} \mathrm{G}^{\omega} L=\mathrm{G}^{\nu+s \omega}$ as ideals of $K[X, Y]$.

Proof. Let $\nu \in \mathbb{N}_{0}^{2 n}$. We can choose a universal Gröbner basis $U$ of $L$ by Theorem 3.8 and we can fix a normal ordering $\preceq \in \mathcal{O}$ by Example 3.2. Thus $U$ is a $\left(\preceq_{\nu}\right)_{\omega}$-Gröbner basis of $L$ for all $\omega \in \Omega$; see Remark 3.9 ,

By Theorem 3.10, $\sigma^{\omega}(U)$ is a ${ }_{\nu}$-Gröbner basis of $\mathrm{G}^{\omega} L$ for all $\omega \in \Omega$. Therefore, by Remark 3.11, $\tau^{\nu}\left(\sigma^{\omega}(U)\right)$ is a $\preceq$-Gröbner basis of $\mathrm{G}^{\nu} \mathrm{G}^{\omega} L$ for all $\omega \in \Omega$. It follows that $\mathrm{G}^{\nu} \mathrm{G}^{\omega} L=\left\langle\tau^{\nu}\left(\sigma^{\omega}(u)\right) \mid u \in U\right\rangle$ for all $\omega \in \Omega$. Putting $s_{\nu}=$ $\max \left\{\operatorname{deg}^{\nu}(u) \mid u \in U, u \neq 0\right\}$ if $U \nsubseteq\{0\}$, and $s_{\nu}=0$ if $U \subseteq\{0\}$, by Lemma 4.7 we get $\mathrm{G}^{\nu} \mathrm{G}^{\omega} L=\left\langle\sigma^{\nu+s \omega}(u) \mid u \in U\right\rangle$ for all $\omega \in \Omega$ and all $s \in \mathbb{N}$ with $s>s_{\nu}$. 
On the other hand, $U$ is a Gröbner basis of $L$ with respect to $\preceq_{\nu+s \omega}$ for all $\omega \in \Omega$ and all $s \in \mathbb{N}$. Therefore, by Theorem 3.10, $\sigma^{\nu+s \omega}(U)$ is a Gröbner basis of $\mathrm{G}^{\nu+s \omega} L$ with respect to $\preceq$, whence $\left\langle\sigma^{\nu+s \omega}(u) \mid u \in U\right\rangle=\mathrm{G}^{\nu+s \omega} L$, for all $\omega \in \Omega$ and all $s \in \mathbb{N}$.

Main Theorem 4.9. Let $M$ be a finitely generated left $W$-module. For all $\nu \in \mathbb{N}_{0}^{2 n}$ there exists $s_{\nu} \in \mathbb{N}_{0}$ with the property that for all $\omega \in \Omega$ and all $s \in \mathbb{N}$ with $s>s_{\nu}$, it holds $\sqrt{ }\left(0: \mathrm{G}^{\nu} \mathrm{G}^{\omega} M\right)=\sqrt{ }\left(0: \mathrm{G}^{\nu} \mathrm{G}^{\nu+s \omega} M\right)=\sqrt{ }\left(0: \mathrm{G}^{\nu+s \omega} M\right)$ as ideals of $K[X, Y]$.

Proof. We fix any $\nu \in \mathbb{N}_{0}^{2 n}$. We find $r \in \mathbb{N}$ such that $M$ is generated over $R$ by $r$ of its elements.

First, by induction over $r$, we prove the existence of $s_{\nu} \in \mathbb{N}_{0}$ such that for all $\omega \in \Omega$ and all $s \in \mathbb{N}$ with $s>s_{\nu}$, it holds $\sqrt{ }\left(0: \mathrm{G}^{\nu} \mathrm{G}^{\omega} M\right)=\sqrt{ }\left(0: \mathrm{G}^{\nu+s \omega} M\right)$.

If $r=1$, then $M \cong W / L$ for a left ideal $L$ of $W$. By Remarks 1.5 and 1.7 and Theorem 4.8 we find $s_{\nu} \in \mathbb{N}_{0}$ such that for all $\omega \in \Omega$ and all $s \in \mathbb{N}$ with $s>s_{\nu}$, it holds $\sqrt{ }\left(0: \mathrm{G}^{\nu} \mathrm{G}^{\omega} W / L\right)=\sqrt{ } \mathrm{G}^{\nu} \mathrm{G}^{\omega} L=\sqrt{ } \mathrm{G}^{\nu+s \omega} L=\sqrt{ }\left(0: \mathrm{G}^{\nu+s \omega} W / L\right)$.

If $r>1$, we find a cyclic submodule $N$ of $M$ such that $P=M / N$ is generated by $r-1$ elements. As before, by Theorem 4.8 we find $s_{\nu}^{\prime} \in \mathbb{N}_{0}$ such that for all $\omega \in \Omega$ and all $s \in \mathbb{N}$ with $s>s_{\nu}^{\prime}$, it holds $\sqrt{ }\left(0: \mathrm{G}^{\nu} \mathrm{G}^{\omega} N\right)=\sqrt{ }\left(0: \mathrm{G}^{\nu+s \omega} N\right)$. By induction we find $s_{\nu}^{\prime \prime} \in \mathbb{N}_{0}$ such that $\sqrt{ }\left(0: G^{\nu} G^{\omega} P\right)=\sqrt{ }\left(0: G^{\nu+s \omega} P\right)$ for all $\omega \in \Omega$ and all $s \in \mathbb{N}$ with $s>s_{\nu}^{\prime \prime}$. By Remark 1.5 we get $\sqrt{ }\left(0: \mathrm{G}^{\nu} \mathrm{G}^{\omega} M\right)=\sqrt{ }\left(0: \mathrm{G}^{\nu} \mathrm{G}^{\omega} N\right) \cap$ $\sqrt{ }\left(0: \mathrm{G}^{\nu} \mathrm{G}^{\omega} P\right)=\sqrt{ }\left(0: \mathrm{G}^{\nu+s \omega} N\right) \cap \sqrt{ }\left(0: \mathrm{G}^{\nu+s \omega} P\right)=\sqrt{ }\left(0: \mathrm{G}^{\nu+s \omega} M\right)$ for all $\omega \in \Omega$ and all $s \in \mathbb{N}$ with $s>s_{\nu}$, where $s_{\nu}=\max \left\{s_{\nu}^{\prime}, s_{\nu}^{\prime \prime}\right\}$, so that $s_{\nu}$ is independent of $\omega$. This completes the induction step.

By Proposition 1.28 and Remarks 1.29 and 1.13, it follows $\sqrt{ }\left(0: \mathrm{G}^{\nu} \mathrm{G}^{\nu+s \omega} M\right)=$ $\sqrt{ } \mathrm{G}^{\nu} \sqrt{ }\left(0: \mathrm{G}^{\nu+s \omega} M\right)=\sqrt{ } \mathrm{G}^{\nu} \sqrt{ }\left(0: \mathrm{G}^{\nu} \mathrm{G}^{\omega} M\right)=\sqrt{ }\left(0: \mathrm{G}^{\nu} \mathrm{G}^{\nu} \mathrm{G}^{\omega} M\right)=\sqrt{ }\left(0: \mathrm{G}^{\nu} \mathrm{G}^{\omega} M\right)$ for all $\omega \in \Omega$ and all $s \in \mathbb{N}$ with $s>s_{\nu}$.

Corollary 4.10. There exists $s_{(1 \mid 1)} \in \mathbb{N}_{0}$ such that for all $\omega \in \Omega$ and all $s \in \mathbb{N}$ with $s>s_{(1 \mid 1)}$ one has $\mathcal{C}^{\omega}(M)=\mathcal{V}^{(1 \mid 1)+s \omega}(M)=\mathcal{C}^{(1 \mid 1)+s \omega}(M)$.

Proof. This is clear by Theorem 4.9 ,

Corollary 4.11. It holds $\mathcal{C}^{(0 \mid 1)}(M)=\mathcal{V}^{(1 \mid s)}(M)=\mathcal{C}^{(1 \mid s)}(M)$ for $s \gg 0$, whereas $\mathcal{C}^{(1 \mid 1)}(M)=\mathcal{V}^{(1 \mid 1)}(M)$.

Proof. The first statement is clear by Corollary 4.10, the second follows from Remark 1.13 .

\section{Application 1: Dimension of characteristic varieties}

In this section, as an application of Theorem 4.9, we aim to furnish a new proof of a classical result: fixing a finitely generated left $W$-module $M$, the characteristic varieties $\mathcal{V}^{\omega}(M), \omega \in \Omega$, all have the same Krull dimension.

This is usually proved, as exposed by Ehlers in [6. Chapter V], by not trivial homological methods. Indeed it turns out that $\operatorname{Kdim}_{K[X, Y]} \mathrm{G}^{\omega} M=2 n-\mathrm{j}_{W}(M)$ for all $\omega \in \Omega$, where $\mathrm{j}_{W}(M)=\inf \left\{i \in \mathbb{N}_{0} \mid \operatorname{Ext}_{W}^{i}(M, W) \neq 0\right\}$.

Bernstein provided a proof in 1971 that $\mathcal{V}^{(1 \mid 1)}(M)$ and $\mathcal{V}^{(0 \mid 1)}(M)$ have the same Krull dimension; see [4, Theorem 3.1]. 
Our proof descends from:

(1) the equality of annihilators obtained in Theorem 4.9, which in particular allows us to pass in a certain sense from non-finite to finite filtrations;

(2) the preservation of the Gelfand-Kirillov dimension when passing from finitely filtered objects to their associated graded objects; see Lemma 5.5

(3) the equality of Krull and Gelfand-Kirillov dimension in the category of noetherian modules over a noetherian commutative $K$-algebra; see Reminder 5.2 .

We begin with some necessary results about the Gelfand-Kirillov dimension that can be found in [11] or [14].

Reminder 5.1. Let $F$ be a field and $B$ be a finitely generated $F$-algebra. We find a generating space of $B$, that is, an $F$-module $V$ of finite length such that $F \subseteq V$ and $B$ is generated as an $F$-algebra by $V$. By $V^{i}, i \in \mathbb{N}_{0}$, we denote the $F$ module consisting of all polynomials in the (generally not commuting) elements of $V$ with coefficients in $F$ of total degree less than or equal to $i$, so that in particular $V^{0}=F, V^{1}=V, V^{i} \subseteq V^{i+1}, B=\bigcup_{i \in \mathbb{N}_{0}} V^{i}$. The Gelfand-Kirillov dimension of $B$ is defined as $\operatorname{GKdim} B=\varlimsup_{i \rightarrow \infty} \log _{i}\left(\operatorname{len}_{F} V^{i}\right) \in[0, \infty]$, and it is independent of $V$. If $A$ is any $F$-algebra, we define $\operatorname{GKdim} A=\sup _{B} \operatorname{GKdim} B$, where the supremum is taken over all finitely generated $F$-subalgebras $B$ of $A$. For finitely generated $F$-algebras, the two definitions are easily shown to be equivalent.

Let $N$ be a finitely generated left $B$-module. We find a generating space of $N$, that is, an $F$-module $W$ of finite length such that $N$ is generated as a $B$ module by $W$. The Gelfand-Kirillov dimension of $N$ is defined as $\operatorname{GKdim}_{B} N=$ $\varlimsup_{i \rightarrow \infty} \log _{i}\left(\operatorname{len}_{F} V^{i} W\right) \in[0, \infty]$, and it is independent of $V$ and of $W$. If $M$ is any $A$-module, we define $\operatorname{GKdim}_{A} M=\sup _{B} \sup _{N} \operatorname{GKdim}_{B} N$, where the suprema are taken over all finitely generated $F$-subalgebras $B$ of $A$ and all finitely generated $B$ submodules of $M$. For finitely generated modules over finitely generated $F$-algebras the two definitions are easily shown to be equivalent.

Reminder 5.2. Let $F$ be a field, $A$ be a finitely generated commutative $F$-algebra, and $M$ be a finitely generated $A$-module. Then for the Krull dimension $\operatorname{Kdim}_{A} M$ of $M$, defined as the supremum of the lengths of chains of prime ideals of the commutative ring $A /(0: M)$, it holds $\operatorname{Kdim}_{A} M=\operatorname{GKdim}_{A} M \in \mathbb{N}_{0} \cup\{-\infty, \infty\}$.

Indeed, in our hypotheses both dimensions are exact (see [11, Theorem 6.14] for the Gelfand-Kirillov dimension), and hence we may assume that $M=A / I$ for some ideal $I$. As both dimensions are preserved when changing the base ring from $A$ to $A / I$ (see [11, Proposition 5.1(c)] for the Gelfand-Kirillov dimension), it is sufficient to compare $\mathrm{K} \operatorname{dim} A / I$ to $\mathrm{GKdim} A / I$. As both dimensions are preserved when passing to integral extensions (see [11, Proposition 5.5] for the Gelfand-Kirillov dimension), by Emmy Noether's Normalization Lemma we may replace the finitely generated $F$-algebra $A / I$ by the polynomial ring $F\left[X_{1}, \ldots, X_{d}\right]$, where $d=\operatorname{Kdim} A / I$. By arguments of linear algebra, one shows that GKdim $F\left[X_{1}, \ldots, X_{d}\right]=d$; for more details see [11, Proposition 7.9] or [3, Corollary 1.1.16].

Alternatively, one easily gets $\operatorname{GKdim} A=\inf \left\{\alpha \in \mathbb{R} \mid \operatorname{len}_{K} V^{i} \leq i^{\alpha}\right.$ for $\left.i \gg 0\right\}$; see [11, Lemma 2.1]. It follows that $\operatorname{GKdim} A$ is indeed equal to the degree of the Hilbert polynomial of $A$, which in turn is equal to $\operatorname{Kdim} A$, and one concludes again by the exactness of both dimensions and by changing the base ring. 
Definition 5.3. Let $F$ be a field, $A$ be an $F$-algebra, $\mathcal{A}$ be a filtration of $A, M$ be a left $A$-module, and $\mathcal{M}$ be an $\mathcal{A}$-filtration of $M$. We say that $\mathcal{M}$ is finite if $\operatorname{len}_{F}\left(\mathrm{~F}_{i} \mathcal{M}\right)<\infty$ for all $i \in \mathbb{Z}$.

Remark 5.4. In the notation of Definition 5.3, if $\mathcal{A}$ is finite and $M$ is finitely generated and $\mathcal{M}$ is good, then $\mathcal{M}$ is finite and discrete. Indeed, $\mathcal{M}$ is equivalent to a standard good filtration $\mathcal{S}$ of $M$; see Proposition 1.24 and Example 1.16. Now, $\mathcal{S}$ is finite whenever $\mathcal{A}$ is finite, and $\mathcal{S}$ is always discrete.

Lemma 5.5. Let $F$ be a field, $A$ be a $K$-algebra, $\mathcal{A}$ be a filtration of $A, M$ be a left $A$-module, and $\mathcal{M}$ be an $\mathcal{A}$-filtration of $M$. Then $\operatorname{GKdim}_{\mathrm{G} \mathcal{A}} \mathrm{G} \mathcal{M} \leq \operatorname{GKdim}_{A} M$.

Furthermore, if the filtration $\mathcal{A}$ is finite and is such that the $F$-algebra GA $\mathcal{A}$ is finitely generated, and if the $\mathcal{A}$-filtration $\mathcal{M}$ is finite and discrete and is such that

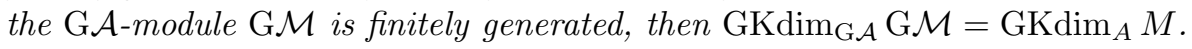

Proof. By arguments of linear algebra, see [11, Lemma 6.5 and Proposition 6.6].

Theorem 5.6. In the notation of the previous section, it holds $\operatorname{Kdim}_{K[X, Y]} \mathrm{G}^{\omega} M=$ $\operatorname{GKdim}_{K[X, Y]} \mathrm{G}^{\omega} M=\operatorname{GKdim}_{W} M$, and hence $\operatorname{Kdim} \mathcal{V}^{\omega}(M)=\operatorname{GKdim}_{W} M$, for all $\omega \in \Omega$.

Proof. Let $\omega \in \Omega$. Since the (1|1)-filtration of $K[X, Y]$ is finite, any good (1|1)filtration of $\mathrm{G}^{\omega} M$ is finite and discrete by Remark 5.4. Thus by Lemma [5.5] $\operatorname{GKdim}_{K[X, Y]} \mathrm{G}^{\omega} M=\mathrm{GKdim}_{K[X, Y]} \mathrm{G}^{(1 \mid 1)} \mathrm{G}^{\omega} M$. By Proposition 1.17, $\mathrm{G}^{(1 \mid 1)} \mathrm{G}^{\omega} M$ is finitely generated over $K[X, Y]$. So, by Reminder [5.2, $\operatorname{GKdim}_{K[X, Y]} \mathrm{G}^{(1 \mid 1)} \mathrm{G}^{\omega} M$ $=\operatorname{GKdim} K[X, Y] / \sqrt{ }\left(0: \mathrm{G}^{(1 \mid 1)} \mathrm{G}^{\omega} M\right)$. Hence by Theorem 4.9, GKdim $K[X, Y] /$ $\sqrt{ }\left(0: \mathrm{G}^{(1 \mid 1)} \mathrm{G}^{\omega} M\right)=\mathrm{GKdim} K[X, Y] / \sqrt{ }\left(0: \mathrm{G}^{(1 \mid 1)+s \omega} M\right), s \gg 0$. Therefore by Reminder 5.2, GKdim $K[X, Y] / \sqrt{ }\left(0: \mathrm{G}^{(1 \mid 1)+s \omega} M\right)=\operatorname{GKdim}_{K[X, Y]} \mathrm{G}^{(1 \mid 1)+s \omega} M$, $s \in \mathbb{N}$. Since the $(1 \mid 1)+s \omega$-filtrations of $W$ are finite, and therefore by Remark 5.4 the good $(1 \mid 1)+s \omega$-filtrations of $M$ are finite and discrete, by Lemma 5.5 and Theorem 2.9 we obtain $\operatorname{GKdim}_{K[X, Y]} \mathrm{G}^{(1 \mid 1)+s \omega} M=\operatorname{GKdim}_{W} M, s \in \mathbb{N}$. As for the Krull dimension, we conclude by Reminder 5.2 .

\section{Application 2: Classification of characteristic varieties}

As before, let $K$ be a field of characteristic 0 . For an arbitrary left ideal $L$ of the first Weyl algebra $W$ over $K$, we aim to classify the characteristic varieties of $W / L$. More precisely, we aim to partition $\Omega=\mathbb{N}_{0}^{2} \backslash\{(0,0)\}$ into regions corresponding to equivalence classes $[\omega]_{\sim_{L}}$ of weights $\omega \in \Omega$ such that $\omega^{\prime} \sim_{L} \omega^{\prime \prime}$ if and only if $\mathrm{G}^{\omega^{\prime}} L=\mathrm{G}^{\omega^{\prime \prime}} L$. This would permit us to determine the number $\chi(L)$ of distinct ideals $\mathrm{G}^{\omega} L, \omega \in \Omega$, which we know to be finite by Corollary 3.12 Hence, because $\mathrm{G}^{\omega^{\prime}} L=\mathrm{G}^{\omega^{\prime \prime}} L$ implies $\mathcal{V}^{\omega^{\prime}}(W / L)=\mathcal{V}^{\omega^{\prime \prime}}(W / L)$ by Remark 1.7 $\chi(L)$ would be an upper bound for the number of distinct $\omega$-characteristic varieties of $W / L$.

We do not succeed in this but, by a computer experiment, we approximate $\Omega / \sim_{L}$, and this allows us to conjecture an upper bound for $\chi(L)$ in terms of total degrees of universal Gröbner bases of $L$.

Remark 6.1. Let $n \in \mathbb{N}$. For each finitely generated left module $M$ over the $n^{\text {th }}$ Weyl algebra over $K$ and for each $\nu \in \mathbb{N}_{0}^{2 n}$ there exists a minimal number $\kappa_{\nu}(M) \in \mathbb{N}_{0}$ such that for all $\omega \in \Omega$ the characteristic varieties $\mathcal{V}^{\nu+s \omega}(M)$ stabilize to $\operatorname{Var}\left(0: \mathrm{G}^{\nu} \mathrm{G}^{\omega} M\right)$ as soon as $s>\kappa_{\nu}(M)$.

In particular, $\mathcal{V}^{(1 \mid 1)+s \omega}(M)$ becomes precisely the critical cone $\mathcal{C}^{\omega}(M)$ for all $\omega \in \Omega$ as soon as $s>\kappa(M)=\kappa_{(1 \mid 1)}(M)$. 
Remark 6.2. Let $n \in \mathbb{N}$. For each left ideal $L$ of the $n^{\text {th }}$ Weyl algebra over $K$ and for each $\nu \in \mathbb{N}_{0}^{2 n}$, we put $\gamma_{\nu}(L)=\inf _{U} \sup _{u \in U \backslash\{0\}} \operatorname{deg}^{\nu}(u)$, where the infimum is taken over all universal Gröbner bases $U$ of $L$. By the proof of Theorem4.8,

(a) $\kappa_{\nu}(W / L) \leq \gamma_{\nu}(L) \in \mathbb{N}_{0}$

(b) $\gamma_{\nu^{\prime}}(L) \leq \gamma_{\nu^{\prime \prime}}(L)$ whenever $\left|\nu^{\prime}\right| \leq\left|\nu^{\prime \prime}\right|$,

(c) $\gamma_{k \nu}(L)=k \gamma_{\nu}(L)$ for all $k \in \mathbb{N}_{0}$.

Experiment 6.3. Let $L$ be any left ideal of the first Weyl algebra $W$ over $K$. By Theorem 4.8 we can compute an approximation of $\Omega / \sim_{L}$ if we know $\kappa_{\nu}(W / L)$ for all $\nu \in \mathbb{N}_{0}^{2 n}$. By the relations (a), (b), (c) of Remark 6.2 we have that $\kappa_{\nu}(W / L) \leq$ $\gamma_{\nu}(L) \leq \gamma_{|\nu|(1 \mid 1)}(L)=|\nu| \gamma(L)$, where we put $\gamma(L)=\gamma_{(1 \mid 1)}(L)$. Therefore, by Theorem 4.8, knowing the upper bound $\gamma(L)$ of $\kappa(W / L)$ is sufficient for computing a (coarser) approximation of $\Omega / \sim_{L}$.

For some numbers $s_{0} \in \mathbb{N}_{0}$ we repeatedly do an experiment parametrized by $s_{0}$ as follows. A computer calculates for us the intersection points among the halflines $\ell_{\nu, \omega} \subseteq \Omega$ of the form $\ell_{\nu, \omega}(s)=\nu+s \omega, \nu \in \mathbb{N}_{0}^{2}, \omega \in \Omega$, for $s>s_{0}$, and it paints incident half-lines with a common colour. The points of $\Omega$ having the same colour turn out to build cones in $\Omega$. For instance, for $s_{0}=3$ the computer program painted seventeen differently coloured cones, among which nine are degenerate, that is, half-lines. For typographical reasons, in Figure 1 we depict the so obtained cones by connected regions in $\mathbb{R}^{2}$, alternately in black and gray. For $s_{0}=3$ the nine degenerate cones are filled in black, whereas the eight non-degenerate cones are filled in gray, and similarly in the other pictures of Figure 1.

By Theorem 4.8, as soon as $s_{0} \geq \gamma(L)$, each of these cones is a subset of precisely one equivalence class of $\Omega / \sim_{L}$. Thus the results of our experiment allow us to conjecture an upper bound for $\chi(L)$ in terms of $\gamma(L)$, namely, $\chi(L) \leq 2^{1+\gamma(L)}+1$.

Our experiment also indicates that the coordinates $\left(x_{1}, x_{2}\right) \in \mathbb{N}_{0}^{2}$ of the vertices of the cones lying in the lower semiquadrant without the diagonal satisfy precisely the conditions

(a) $F(1) \leq x_{1} \leq F\left(2+s_{0}\right)$,

(b) $F(0) \leq x_{2} \leq F\left(1+s_{0}\right)$,

(c) $\operatorname{gcd}\left(x_{1}, x_{2}\right)=1$, and

(d) $x_{1}>x_{2}$,

where $F(s)$ is the $s^{\text {th }}$ Fibonacci number, that is, $F(0)=0, F(1)=1$, and $F(s)=$ $F(s-1)+F(s-2)$ for all $s \geq 2$. For instance, if $s_{0}=3$, these coordinates are $(1,0),(2,1),(3,1),(4,1),(3,2),(5,2),(4,3),(5,3)$.

So $2^{\gamma(L)}$ is equal to the number of the points $\left(x_{1}, x_{2}\right) \in \mathbb{N}_{0}^{2}$ satisfying the conditions (a)-(d) with $s_{0}=\gamma(L)$, and the experiment indicates that $\chi(L) \leq$ $\#\left\{\left(x_{\sigma(1)}, x_{\sigma(2)}\right) \in \mathbb{N}_{0}^{2} \mid \sigma \in \Sigma_{2} \wedge F(1) \leq x_{1} \leq F(2+\gamma(L)) \wedge F(0) \leq x_{2} \leq F(1+\gamma(L))\right.$ $\left.\wedge \operatorname{gcd}\left(x_{1}, x_{2}\right)=1 \wedge x_{1} \geq x_{2}\right\}=\# \Sigma_{2} \cdot\left(2^{\gamma(L)}+1\right)-\left(\# \Sigma_{2}-1\right)=2^{1+\gamma(L)}+1$, where $\Sigma_{2}$ is the second symmetric group.

Remark 6.4. Weyl algebras are the prototype of algebras of solvable type ( see [10]), and as in the polynomial case a universal Gröbner basis of $L$ can be constructed as a union of reduced Gröbner bases of $L$. In [1, Corollary 0.2], an upper bound is given for the total degree of elements of reduced Gröbner bases of a left ideal of an algebra of solvable type in terms of the total degree of generators of the ideal, thus in particular an upper bound for $\gamma(L)$. Therefore if our conjecture is true, one obtains an upper bound for the cardinality of $\Omega / \sim_{L}$ in terms of the total degree of generators of $L$. 


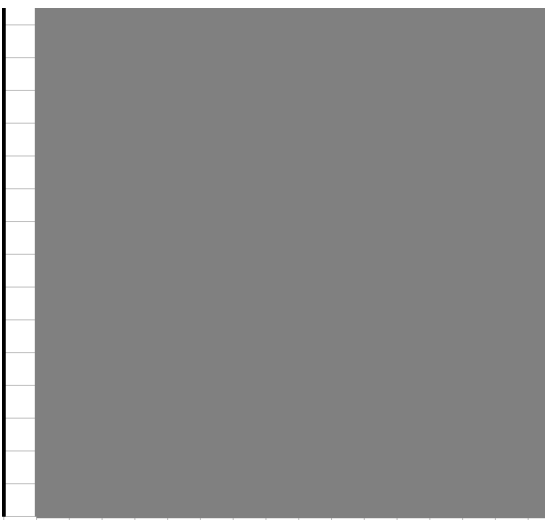

(a) $s_{0}=0$

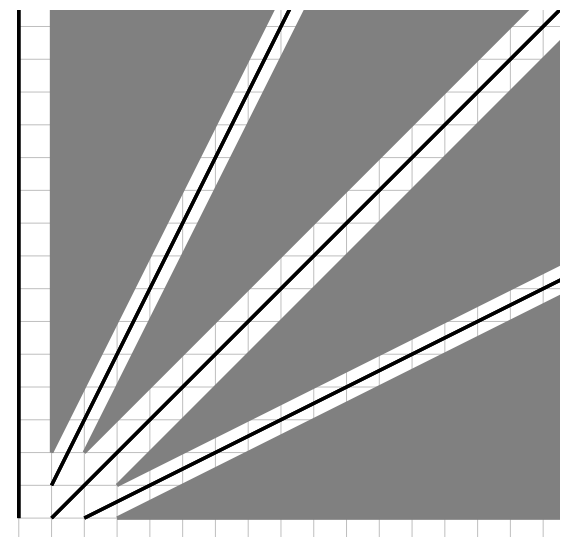

(c) $s_{0}=2$

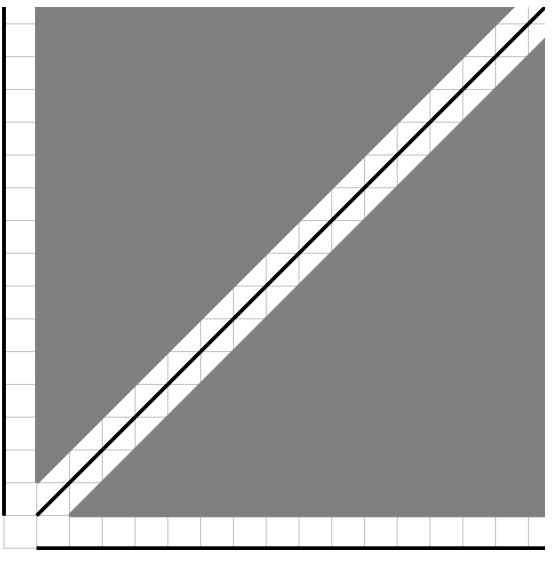

(b) $s_{0}=1$

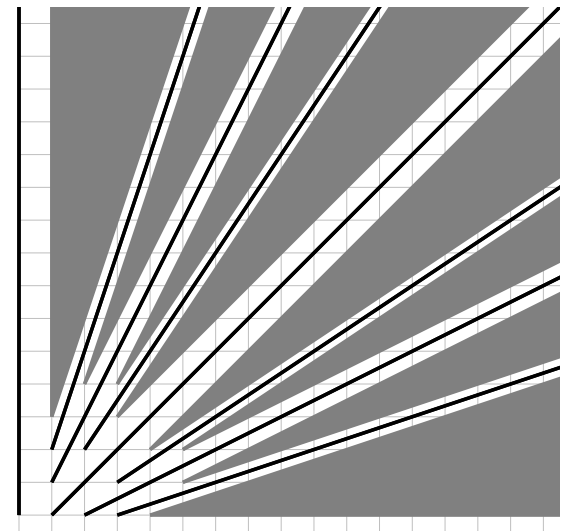

(d) $s_{0}=3$

FIgURE 1. Equality regions of characteristic varieties

Question 6.5. We may ask whether similar upper bounds for $\chi(L)$ as in Experiment 6.3 exist when considering a left ideal $L$ of the $n^{\text {th }}$ Weyl algebra for $n>1$, namely, (1) a bound in terms of $n$ and $\gamma(L)$, and (2) a bound in terms of Fibonacci numbers.

\section{REFERENCES}

1. M. Aschenbrenner, A. Leykin, Degree Bounds for Gröbner Bases in Algebras of Solvable Type, J. of Pure and Applied Alg. 213 (2009), 1578-1605. MR2517995 (2010g:13042)

2. M. Assi, F. J. Castro-Jiménez, M. Granger, The Gröbner Fan of an $A_{n}$-module, J. of Pure and Applied Alg. 150 (2000), 27-39. MR1762918 (2001j:16036)

3. J. P. Bell, Affine Rings of Low GK Dimension, Dissertation, UCSD, 2002. MR2703199

4. J. N. Bernstein, Modules over a Ring of Differential Operators. Study of the Fundamental Solutions of Equations with Constant Coefficients, translated from the Russian original, Funktsional'nyi Analiz i Ego Prilozheniya 5(2) (1971), 1-16. MR0290097 (44:7282)

5. R. Boldini, Finiteness of Leading Monomial Ideals and Critical Cones of Characteristic Varieties, Ph.D. Dissertation, UZH, 2012. 
6. A. Borel et al., Algebraic D-Modules, Perspectives in Mathematics 2, Academic Press, 1987. MR 882000 (89g:32014)

7. M. Brodmann, Algebren von Differentialoperatoren, Lecture Notes, UZH, 1986.

8. S. C. Coutinho, A Primer of Algebraic D-Modules, LMS Student Texts 33, Cambridge University Press, 1995. MR 1356713 (96j:32011)

9. D. Cox, J. Little, D. O'Shea, Ideals, Varieties, and Algorithms, Undergraduate Texts in Mathematics, Second Edition, Springer, 1997. MR,1417938 (97h:13024)

10. A. Kandry-Rody, V. Weispfenning, Non-commutative Gröbner Bases in Algebras of Solvable Type, J. of Symb. Comp. 9 (1990), 1-26. MR.1044911 (91e:13025)

11. G. R. Krause, T. H. Lenagan, Growth of Algebras and Gelfand-Kirillov Dimension, Graduate Studies in Mathematics 22, AMS, 2000. MR.1721834 (2000j:16035)

12. H. Li, Noncommutative Gröbner Bases and Filtered-Graded Transfer, Lecture Notes in Mathematics 1795, Springer, 2002. MR 1947291 (2003i:16065)

13. H. Li, F. van Oystaeyen, Zariskian Filtrations, K-Monographs in Mathematics 2, Kluwer Academic Publishers, 1996. MR.1420862 (97m:16083)

14. J. C. McConnell, J. C. Robson, Noncommutative Noetherian Rings, Wiley, 1987. MR934572 (89j:16023)

15. M. Saito, B. Sturmfels, N. Takayama, Gröbner Deformations of Hypergeometric Differential Equations, Algorithms and Computation in Mathematics 6, Springer, 2000. MR 1734566 (2001i:13036)

16. B. Sturmfels, Gröbner Bases and Convex Polytopes, University Lecture Series 8, AMS, 1996. MR.1363949 (97b:13034)

17. V. Weispfenning, Constructing Universal Gröbner Bases, Lecture Notes in Computer Sciences 356, 408-417, Springer, 1989. MR1008554 (91e:13029)

Institut FÜr Mathematik, Universität Zürich, Winterthurerstr. 190, CH-8057 ZÜRICH, SWITZERLAND

E-mail address: roberto.boldini@math.uzh.ch 